\title{
THE FISHER-KPP EQUATION WITH NONLINEAR FRACTIONAL DIFFUSION*
}

\author{
DIANA STAN ${ }^{\dagger}$ AND JUAN LUIS VÁZQUEZ ${ }^{\dagger}$
}

\begin{abstract}
We study the propagation properties of nonnegative and bounded solutions of the class of reaction-diffusion equations with nonlinear fractional diffusion: $u_{t}+(-\Delta)^{s}\left(u^{m}\right)=f(u)$. For all $0<s<1$ and $m>m_{c}=(N-2 s)_{+} / N$, we consider the solution of the initial-value problem with initial data having fast decay at infinity and prove that its level sets propagate exponentially fast in time, in contrast to the traveling wave behavior of the standard KPP case, which corresponds to putting $s=1, m=1$, and $f(u)=u(1-u)$. The proof of this fact uses as an essential ingredient the recently established decay properties of the self-similar solutions of the purely diffusive equation, $u_{t}+(-\Delta)^{s} u^{m}=0$.
\end{abstract}

Key words. reaction-diffusion equation, Fisher-KPP equation, propagation of level sets, nonlinear fractional diffusion

AMS subject classifications. 35K57, 26A33, 35K65, 76S05, 35C06, 35C07

DOI. $10.1137 / 130918289$

1. Introduction. We consider the following reaction-diffusion problem:

$$
\begin{cases}u_{t}(x, t)+L_{s} u^{m}(x, t)=f(u) & \text { for } x \in \mathbb{R}^{N} \text { and } t>0, \\ u(x, 0)=u_{0}(x) & \text { for } x \in \mathbb{R}^{N},\end{cases}
$$

where $L_{s}=(-\Delta)^{s}$ is the fractional Laplacian operator with $s \in(0,1)$. We are interested in studying the propagation properties of nonnegative and bounded solutions of this problem in the spirit of the Fisher-KPP theory. Therefore, we assume that the reaction term $f(u)$ satisfies

$$
f \in C^{1}([0,1]) \text { is a concave function with } f(0)=f(1)=0, f^{\prime}(1)<0<f^{\prime}(0) .
$$

For example, we can take $f(u)=u(1-u)$. Our results will depend on the parameters $m$ and $s$, according to the ranges $m_{c}<m<m_{1}, m_{1}<m \leq 1$, and $m>1$, where

$$
m_{c}=\frac{(N-2 s)_{+}}{N}, \quad m_{1}=\frac{N}{N+2 s} .
$$

1.1. Perspective. The traveling wave behavior. The problem with standard diffusion goes back to the work of Kolmogorov, Petrovskii, and Piskunov [23], which presents the most simple reaction-diffusion equation concerning the concentration $u$ of a single substance in one spatial dimension,

$$
\partial_{t} u=D u_{x x}+f(u) .
$$

The choice $f(u)=u(1-u)$ yields Fisher's equation [20], which was originally used to describe the spreading of biological populations. The celebrated result says that

\footnotetext{
*Received by the editors April 23, 2013; accepted for publication (in revised form) June 11, 2014; published electronically September 25, 2014. This research was supported by the Spanish Project MTM2011-24696.

http://www.siam.org/journals/sima/46-5/91828.html

${ }_{\dagger}^{\dagger}$ Departamento de Matemáticas, Universidad Autónoma de Madrid, 28049 Madrid, Spain (diana.stan@uam.es, juanluis.vazquez@uam.es).
} 
the long-time behavior of any solution of (1.3), with suitable data $0 \leq u_{0}(x) \leq 1$ that decay fast at infinity, resembles a traveling wave with a definite speed. When considering (1.3) in dimensions $N \geq 1$, the problem becomes

$$
u_{t}-\Delta u=f(u) \quad \text { in }(0,+\infty) \times \mathbb{R}^{N},
$$

which corresponds to (1.1) in the case when $L_{s}=-\Delta$, the standard Laplacian. This case has been studied by Aronson and Weinberger in [3, 4], where they prove the following result.

THEOREM AW. Let $u$ be a solution of (1.4) with $u_{0} \neq 0$ compactly supported in $\mathbb{R}^{N}$ and satisfying $0 \leq u_{0}(\cdot) \leq 1$. Let $c_{*}=2 \sqrt{f^{\prime}(0)}$. Then,

1. if $c>c_{*}$, then $u(x, t) \rightarrow 0$ uniformly in $\{|x| \geq c t\}$ as $t \rightarrow \infty$;

2. if $c<c_{*}$, then $u(x, t) \rightarrow 1$ uniformly in $\{|x| \leq c t\}$ as $t \rightarrow \infty$.

In addition, problem (1.4) admits planar traveling wave solutions connecting 0 and 1 , that is, solutions of the form $u(x, t)=\phi(x \cdot e+c t)$ with

$$
-\phi^{\prime \prime}+c \phi^{\prime}=f(\phi) \text { in } \mathbb{R}, \quad \phi(-\infty)=0, \phi(+\infty)=1 .
$$

This asymptotic traveling wave behavior has been generalized in many interesting ways. Of concern here is the consideration of nonlinear diffusion. De Pablo and Vázquez study in [17] the existence of traveling wave solutions and the property of finite propagation for the reaction-diffusion equation

$$
u_{t}=\left(u^{m}\right)_{x x}+\lambda u^{n}(1-u), \quad(x, t) \in \mathbb{R} \times(0, \infty)
$$

with $m>1, \lambda>0, n \in \mathbb{R}$, and $u=u(x, t) \geq 0$. Similar results hold also for other slow diffusion cases, $m>1$, studied by de Pablo and Sánchez [16].

1.2. Nontraveling wave behavior. Departing from these results, King and McCabe examined in [22] a case of fast diffusion, namely,

$$
u_{t}=\Delta u^{m}+u(1-u), \quad x \in \mathbb{R}^{N}, t>0,
$$

where $(N-2)_{+} / N<m<1$. They showed that the problem does not admit traveling wave solutions. Using a detailed formal analysis, they also showed that level sets of the solutions of the initial-value problem with suitable initial data propagate exponentially fast in time. They extended the results to all $0<m<1$.

On the other hand, and independently, Cabré and Roquejoffre in [11, 12] studied the case of fractional linear diffusion, $s \in(0,1)$ and $m=1$, and they concluded in the same vein that there is no traveling wave behavior as $t \rightarrow \infty$, and indeed the level sets propagate exponentially fast in time. The fast propagation is not surprising because of the long-distance dispersal, even if the diffusion is linear.

Motivated by these two examples of break of the asymptotic traveling wave structure, we study here the case of a diffusion that is both fractional and nonlinear, namely, problem (1.1) in the range $s \in(0,1)$ and $m>m_{c}$. The initial datum $u_{0}(x): \mathbb{R}^{N} \rightarrow[0,1]$ and satisfies a growth condition of the form

$$
0 \leq u_{0}(x) \leq C|x|^{-\lambda(N, s, m)} \quad \forall x \in \mathbb{R}^{N},
$$

where the exponent $\lambda(N, s, m)$ is stated explicitly in the different ranges, $m_{c}<m<$ $m_{1}$ and $m_{1}<m$. In this paper we establish the negative result about traveling wave behavior, more precisely, we prove that an exponential rate of propagation of level 
sets is true in all cases. We also explain the mechanism for it in simple terms: the exponential rate of propagation of the level sets of solutions (with initial data having a certain minimum decay for large $|x|$ ) is a consequence of the power-like decay behavior of the fundamental solutions of the diffusion problem studied in [26]. Therefore, we obtain two main cases in the analysis, $m_{c}<m<m_{1}$ and $m>m_{1}$, depending on that behaviour.

1.3. Main results. The existence of a unique mild solution of problem (1.1) follows by semigroup approach. The mild solution corresponding to an initial datum $u_{0} \in L^{1}\left(\mathbb{R}^{N}\right), 0 \leq u_{0} \leq 1$, is in fact a positive, bounded, strong solution with classical regularity. In the appendix we give a brief discussion of these properties. Let us introduce some notation. Throughout the paper we will consider $m>m_{c}$. Once and for all, we put

$$
\begin{aligned}
\beta & =1 /(N(m-1)+2 s), \\
\sigma_{1} & =\frac{1-m}{2 s} f^{\prime}(0), \quad \sigma_{2}=\frac{1}{N+2 s} f^{\prime}(0), \quad \sigma_{3}=\frac{1+2(m-1) \beta s}{N+2 s} f^{\prime}(0) .
\end{aligned}
$$

The value $\sigma_{1}$ appears for $m_{c}<m<m_{1}$ and then $\sigma_{1}>\sigma_{2}$. Notice also that $\sigma_{2}<\sigma_{3}$ for $m>1$. Here is the precise statement of our main results for the solutions of the generalized KPP problem (1.1).

Theorem 1.1. Let $N \geq 1, s \in(0,1)$, $f$ satisfying (1.2) and $m_{1}<m \leq 1$. Let $u$ be a solution of (1.1), where $0 \leq u_{0}(\cdot) \leq 1$ is measurable, $u_{0} \neq 0$, and satisfies

$$
0 \leq u_{0}(x) \leq C|x|^{-(N+2 s)} \quad \forall x \in \mathbb{R}^{N} .
$$

Then

1. if $\sigma>\sigma_{2}$, then $u(x, t) \rightarrow 0$ uniformly in $\left\{|x| \geq e^{\sigma t}\right\}$ as $t \rightarrow \infty$;

2. if $\sigma<\sigma_{2}$, then $u(x, t) \rightarrow 1$ uniformly in $\left\{|x| \leq e^{\sigma t}\right\}$ as $t \rightarrow \infty$.

TheOrem 1.2. Let $N \geq 1, s \in(0,1)$, $f$ satisfying (1.2) and $m_{c}<m<m_{1}$. Let $u$ be a solution of $(1.1)$, where $0 \leq u_{0}(\cdot) \leq 1$ is measurable, $u_{0} \neq 0$, and satisfies

$$
0 \leq u_{0}(x) \leq C|x|^{-2 s /(1-m)} \quad \forall x \in \mathbb{R}^{N} .
$$

Then

1. if $\sigma>\sigma_{1}$, then $u(x, t) \rightarrow 0$ uniformly in $\left\{|x| \geq e^{\sigma t}\right\}$ as $t \rightarrow \infty$;

2. if $\sigma<\sigma_{1}$, then $u(x, t) \rightarrow 1$ uniformly in $\left\{|x| \leq e^{\sigma t}\right\}$ as $t \rightarrow \infty$.

Theorem 1.3. Let $N \geq 1, s \in(0,1)$, $f$ satisfying (1.2) and $m>1$. Let $u$ be a solution of (1.1), where $0 \leq u_{0}(\cdot) \leq 1$ is measurable, $u_{0} \neq 0$, and satisfies

$$
0 \leq u_{0}(x) \leq C|x|^{-(N+2 s)} \quad \forall x \in \mathbb{R}^{N} .
$$

Then

1. if $\sigma>\sigma_{3}$, then $u(x, t) \rightarrow 0$ uniformly in $\left\{|x| \geq e^{\sigma t}\right\}$ as $t \rightarrow \infty$;

2. if $\sigma<\sigma_{2}$, then $u(x, t) \rightarrow 1$ uniformly in $\left\{|x| \leq e^{\sigma t}\right\}$ as $t \rightarrow \infty$.

Remarks. In all ranges of parameters $m>m_{c}$, there appear critical values of $\sigma$ with an influence on the behavior of the level sets.

- In the case $m_{1}<m<1$, the case $\sigma=\sigma_{2}$ is still open. This critical exponent is the same as in the case of the linear diffusion $m=1$, proved in [12].

- In the range $m_{c}<m<m_{1}$, the case $\sigma=\sigma_{1}$ is still open. In particular, for the classical case $s=1$ and $f(u)=u(1-u)$ we get $\sigma_{1}=\frac{1-m}{2}$, which is a critical speed found by King and McCabe [22]. In this way, we complete their result with rigorous proofs to all $s \in(0,1)$.

Copyright (C) by SIAM. Unauthorized reproduction of this article is prohibited. 


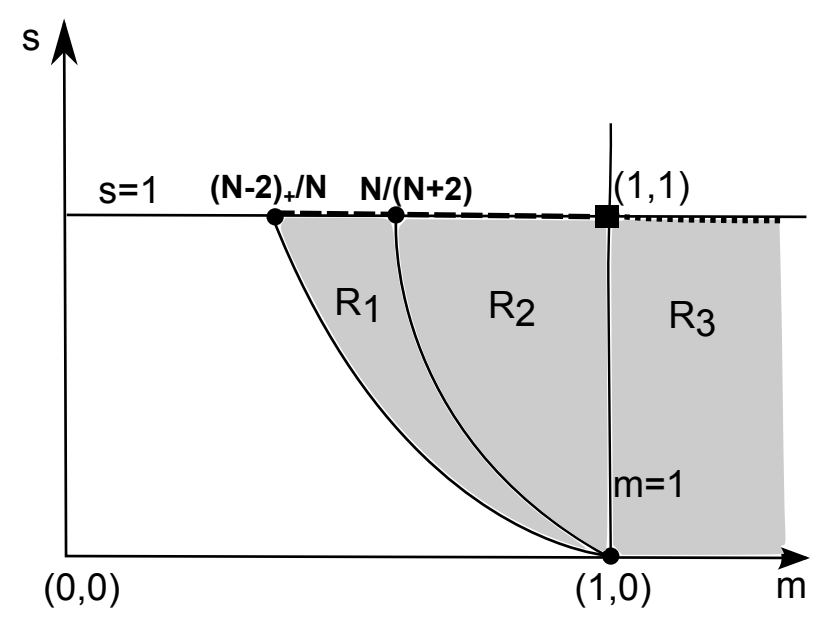

FIG. 1. Ranges of parameters $m$ and $s$ : we study the cases $R_{1}=\left\{s \in(0,1),(N-2 s)_{+} / N<\right.$ $m<N /(N+2 s)\}, R_{2}=\{s \in(0,1), N /(N+2 s)<m \leq 1\}, R_{3}=\{s \in(0,1), m>1\}$.

- In the case $m>1$, we do not cover the entire interval $\left[\sigma_{2}, \sigma_{3}\right]$. If we could prove that the behavior in this interval is the same as in the case $\sigma>\sigma_{3}$, then the results of Theorems 1.1 and 1.3 would agree.

- The result of Theorems 1.1 and 1.2 is true also in the case $m=m_{1}$, where $\sigma_{1}=\sigma_{2}$. The outline of the proof is the same, but there are a number of additional technical difficulties, typical of borderline cases. We have decided to skip the lengthy analysis of this case because of the lack of novelty for our intended purpose.

Our main conclusion is that exponential propagation is shown to be the common occurrence, and the existence of traveling wave behavior is reduced to the classical KPP cases mentioned at the beginning of this discussion (see the dotted line in Figure 1).

As we have mentioned, one of the motivations of the work was to make clear the mechanism that explains the exponential rate of expansion in simple terms, even in this situation that is more complicated than [12]. In fact, due to the nonlinearity, the solution of the diffusion problems involved in the proofs does not admit an integral representation as the case $m=1$. Instead, we will use as an essential tool the behavior of the fundamental solution of the fractional porous medium equation (FPME), also called the Barenblatt solution, recently studied in [26]. To be precise, the decay rate of the tail of these solutions as $|x| \rightarrow \infty$ is the essential information we use to calculate the rates of expansion. This information is combined with more or less usual techniques of linearization and comparison with sub- and supersolutions. We also need accurate lower estimates for positive solutions of the FPME and a further self-similar analysis for the linear diffusion problem.

1.4. Organization of the proofs. In section 4 , under the assumption of initial datum with the decay (1.5), we prove convergence to 0 in the outer set $\left\{|x| \geq e^{\sigma t}\right\}$ by constructing a supersolution of the linearized problem with reaction term $f^{\prime}(0) u$. The arguments hold for $\sigma$ larger than the corresponding critical velocity.

In section 5 we prove convergence to 1 on the inner sets $\left\{|x| \leq e^{\sigma t}\right\}$ in various steps. We only assume $0 \leq u_{0} \leq 1, u_{0} \neq 0$. We first show that the solution reaches a certain minimum profile for positive times, thanks to the analysis of Theorem 1.4 
below; we then perform an iterative proof the conservation in time of this minimum level, and finally convergence to 1 is obtained by constructing a supersolution to the problem satisfied by $1-u^{m}$. Therefore, we deal with a problem of the form

$$
a(x, t) w_{t}(x, t)+L_{s} w(x, t)+b_{0} w(x, t) \geq 0 .
$$

A suitable choice for constructing the supersolution $w$ is represented by self-similar solutions of the form $U(x, t)=t^{\alpha^{\prime}} F\left(|x| t^{-\beta^{\prime}}\right)$ of the linear problem

$$
U_{t}+L_{s} U=0
$$

with radial increasing initial data. This motivates us to derive a number of properties of the linear diffusion problem (1.9), also known as the fractional heat equation. In particular, we need to show that the profile $F$ mentioned above has the same asymptotic behavior as the initial data. In order to establish such a fact we have to review (section 6) the properties of the fundamental solution of problem (1.9):

$$
K_{s}(x, t)=t^{-\frac{N}{2 s}} f\left(t^{-\frac{1}{2 s}}|x|\right), \quad f(r) \sim r^{-(N+2 s)} .
$$

We perform a further analysis of the profile $f$ by proving that $r f^{\prime} \sim r^{-(N+2 s)}$.

Remark. As a consequence of the exponential propagation of the level sets, we immediately obtain the nonexistence of traveling wave solutions of the form $u(x, t)=$ $\varphi(x+t \cdot e)$. However, our results amount to the existence of a kind of logarithmic traveling wave behavior, that is, a kind of wave solutions that travel linearly if we measure distance in a logarithmic scale. This whole issue deserves further investigation.

1.5. New estimates for the fractional diffusion problem. The study of the sub- and supersolutions is strongly determined by the existence of suitable lower parabolic estimates for the associated diffusion problem, the FPME

$$
\begin{cases}\underline{u}_{t}(x, t)+L_{s} \underline{u}^{m}(x, t)=0 & \text { for } x \in \mathbb{R}^{N} \text { and } t>0, \\ \underline{u}(x, 0)=\underline{u}_{0}(x) & \text { for } x \in \mathbb{R}^{N} .\end{cases}
$$

In section 3 , we devote a separate study to the case $m>1$ of the behavior of the solution when $|x| \rightarrow \infty$, more precisely, its rate of decay, for small times $t>0$. Our main result says that roughly speaking

$$
\underline{u}(x, t) \sim t|x|^{-(N+2 s)}
$$

when $|x|$ is large and $t$ small. The precise result is as follows.

TheOREM 1.4. Let $m>1$. Let $\underline{u}(x, t)$ be a solution of problem (1.10) with initial data $u_{0}(x) \geq 0$ such that $u_{0}(x) \geq 1$ in the ball $B_{1}(0)$. Then there is a time $t_{1}>0$ and constants $C_{*}, R>0$ such that

$$
\underline{u}(x, t) \geq C_{*} t|x|^{-(N+2 s)}
$$

if $|x| \geq R$ and $0<t<t_{1}$.

The fact that solutions of the FPME with nonnegative initial data become immediately positive for all times $t>0$ in the whole space has been proved in $[13,14]$. Such result is true not only for $0<s<1$ and $m>1$, but also for $0<s<1$ and $m>m_{c}=(N-2 s)_{+} / N$, this lower restriction on $m$ aimed at avoiding the possibility of extinction in finite time.

Copyright (C) by SIAM. Unauthorized reproduction of this article is prohibited. 
Precise quantitative estimates of positivity for $t>0$ on bounded domains of $\mathbb{R}^{N}$ have been obtained in the recent paper [9]. The estimates of that reference are also precise in describing the behavior as $|x| \rightarrow \infty$ when $m<1$ (fast diffusion), but they are not relevant to establishing the far-field behavior for $m>1$. We recall that with the limit $s \rightarrow 1$ with $m>1$ fixed we get the standard porous medium equation, where positivity at infinity for all nonnegative solutions is false due to the property of finite propagation; cf. [27]. This explains that some special characteristic of fractional diffusion must play a role if positivity is true.

1.6. Remarks on the reaction problem. (a) As further evidence of the influence of the tail of the data on the propagation rate, we consider the purely reactive problem (no diffusion)

$$
u_{t}=f(u), \quad x \in \mathbb{R}^{N}, t>0,
$$

with initial datum $u_{0}$ and $f(u) \sim u(1-u) \sim f^{\prime}(0) u$ as $u \rightarrow 0$. It is easy to see that when we simplify $f(u)$ to $f^{\prime}(0) u=a u$, the exact solution is

$$
u(x, t)=u_{0} e^{f^{\prime}(0) t} .
$$

Let us examine the level sets in two particular cases.

(b) Exponential decay. By considering initial datum of the form $u_{0}(x) \sim e^{-x^{2}}$ for large $|x|$, then the solution $u(x, t)$ satisfies a similar behavior

$$
u(x, t) \sim e^{-\left(x^{2}-a t\right)} \text { for large } x .
$$

The level sets $u(x, t)=$ constant are characterized by $x=\sqrt{a t+c}$.

(c) Power decay. By considering initial datum of the form $u_{0}(x) \sim|x|^{-(N+2 s)}$ for large $|x|$, then the solution $u(x, t)$ is such that

$$
u(x, t) \sim e^{a t}|x|^{-(N+2 s)} .
$$

The level sets $u(x, t)=$ constant are characterized by $|x| \sim e^{\frac{a}{N+2 s} t}$.

(d) Conclusion. For $|x|$ large, the solution of the reaction-diffusion problem (1.1) behaves like the solution of problem (1.12), that is, the nondiffusion case. The fractional diffusion term $(-\Delta)^{s} u^{m}$ does not change the basic behavior of the solution for large $|x|$. This fact has also been observed by King and McCabe in [22] in the fast diffusion case with the standard Laplace operator.

1.7. Comment on applications and mathematical motivation. Anomalous diffusion processes with long-range effects connected to Levy flights in stochastic processes are usually modeled with nonlocal operators, in particular with the fractional Laplacian. They describe different phenomena in physics, finance, biology, and many others. Equations involving anomalous diffusion may take a nonlinear form. (See [9] for a more detailed summary.)

The reaction-diffusion problem (1.1) with linear diffusion $m=1$, recently studied by Cabré and Roquejoffre [12], appears in population dynamics (see [6]). The nonlocal character of the diffusion operator generates the following event: the stable state $u=1$ invades faster (with exponential speed) the unstable state $u=0$. This behavior was seen already in the case of local nonlinear diffusion with nonlinearity $m<1$ (fast diffusion) by King and McCabe [22]. The study of the problem involving nonlinear fractional diffusion and KPP reaction is motivated by such preceding works. We show 
that the exponential invasion of the unstable state by the stable one is a quite general phenomenon that holds for a wide range of equations combining nonlinearity and KPP reaction. As a conclusion, the traveling wave with constant speed of the original KPP model looks in that respect like a very special phenomenon.

\section{Preliminaries.}

2.1. Nonlinear diffusion. The FPME. We recall some useful results concerning the porous medium equation with fractional diffusion (FPME). We refer to [14], where the authors develop the basic theory for the general problem

$$
\begin{cases}u_{t}=-L_{s}\left(|u|^{m-1} u\right) & \text { for } x \in \mathbb{R}^{N} \text { and } t>0, \\ u(0, x)=u_{0}(x) & \text { for } x \in \mathbb{R}^{N},\end{cases}
$$

with data $u_{0} \in L^{1}\left(\mathbb{R}^{N}\right)$ and exponents $0<s<1$ and $m>0$. Existence and uniqueness of a weak solution is established for $m>m_{c}=(N-2 s)_{+} / N$ giving rise to an $L^{1}$-contraction semigroup. Recently in [15], the classical regularity was proved. Positivity of the solution for any $m>0$ corresponding to nonnegative data has been proved in [9]. We give a brief discussion on these facts in the appendix.

2.2. Barenblatt solutions of the FPME. An important tool that we use in the paper is represented by the so-called Barenblatt solutions of the FPME. In [26], Vázquez proves existence, uniqueness, and main properties of such fundamental solutions of the equation

$$
u_{t}+(-\Delta)^{s} u^{m}=0,
$$

taking as initial data a Dirac delta $u(x, 0)=M \delta(x)$, where $M>0$ is the mass of the solution. We will give here a short description of these functions and recall their main properties we need in the paper. Next, we recall Theorem 1.1 from [26].

TheOrem 2.1. For every choice of parameters $s \in(0,1)$ and $m>m_{c}=$ $\max \{(N-2 s) / N, 0\}$, and every $M>0,(2.2)$ admits a unique fundamental solution with initial condition $M \delta(x)$; it is a nonnegative and continuous weak solution for $t>0$ and takes the initial data in the sense of Radon measures. Such solution has the self-similar form

$$
B_{M}(x, t)=t^{-\alpha} F_{M}\left(|x| t^{-\beta}\right)
$$

for suitable $\alpha$ and $\beta$ that can be calculated in terms of $N$ and $s$ in a dimensional way, precisely

$$
\alpha=\frac{N}{N(m-1)+2 s}, \quad \beta=\frac{1}{N(m-1)+2 s} .
$$

The profile function $F_{M}(r), r \geq 0$, is a bounded and Hölder continuous function, it is positive everywhere, it is monotone, and it goes to zero at infinity.

In what follows we denote by $F_{M}$ the profile corresponding to the Barenblatt soluton with mass $M$, as stated in the above theorem. By Theorem 2.1 there exists a unique self-similar solution $B_{1}(x, t)$ with mass $M=1$ of problem (2.2) and, moreover, it has the form $B_{1}(x, t)=t^{-\alpha} F_{1}\left(|x| t^{-\beta}\right)$. Let $B_{M}(x, t)$ the unique self-similar solution of problem (2.2) with mass $M$. Such function will be of the form

$$
B_{M}(x, t)=M B_{1}\left(x, M^{m-1} t\right),
$$

Copyright (C) by SIAM. Unauthorized reproduction of this article is prohibited. 
which can be written in terms of the profile $F_{1}$ as

$$
B_{M}(x, t)=M^{1-(m-1) \alpha} t^{-\alpha} F_{1}\left(\left(M_{1}^{m-1} t\right)^{-\beta}|x|\right) .
$$

Moreover, the precise characterization of the profile $F_{M}$ is given by Theorem 8.1 of $[26]$.

TheOrem 2.2. For every $m>m_{1}=N /(N+2 s)$ we have the asymptotic estimate

$$
\lim _{r \rightarrow \infty} F_{M}(r) r^{N+2 s}=C_{1} M^{\sigma}
$$

where $M=\int F(x) d x, C_{1}=C_{1}(m, N, s)>0$ and $\sigma=\left(m-m_{1}\right)(N+2 s) \beta$. On the other hand, for $m_{c}<m<m_{1}$, there is a constant $C_{\infty}(m, N, s)$ such that

$$
\lim _{r \rightarrow \infty} F_{M}(r) r^{2 s /(1-m)}=C_{\infty}
$$

The case $m=m_{1}$ has a logarithmic correction. The profile $F_{M}$ has the upper bound

$$
F_{M}(r) \leq C r^{-N-2 s+\epsilon} \quad \forall r>0
$$

for every $\epsilon>0$ and the lower bound

$$
F_{M}(r) \geq C r^{-N-2 s} \log r \quad \forall \text { large } r .
$$

We state now some properties of the profile $F_{M}(r), r \geq 0$, obtained as consequences of formula (2.5) that we will use in what follows. Let us consider first the case $m>m_{1}$.

1. $F_{1}$ attains its maximum when $r=0$, i.e., $F_{M}(r) \leq F_{M}(0)$, for all $r \geq 0$.

2. There exists $K_{1}>0$ such that

$$
F_{M}(r) \leq K_{1} r^{-(N+2 s)} \quad \forall r>0 .
$$

3. There exists $K_{2}>0$ such that

$$
F_{M}(r) \geq K_{2}\left(1+r^{N+2 s}\right)^{-1} \quad \forall r \geq 0 .
$$

Similar estimates hold also in the case $m_{c}<m<m_{1}$, and the corresponding tail behavior is different, $F_{M}(r) \sim r^{-2 s /(1-m)}$. This will have an effect in the different results we get for the generalized KPP problem.

As a consequence, Vázquez also proves that the asymptotic behavior of general solutions of problem (2.1) is represented by such special solutions as described in Theorem 10.1 from [26].

TheOrEm 2.3. Let $u_{0}=\mu \in \mathcal{M}_{+}\left(\mathbb{R}^{N}\right), M=\mu\left(\mathbb{R}^{N}\right)$ and let $u$ be the solution of (2.1) and $B_{M}$ be the self-similar Barenblatt solution with mass $M$. Then we have

$$
\lim _{t \rightarrow \infty}\left|u(x, t)-B_{M}(x, t ; M)\right|=0
$$

and the convergence is uniform in $\mathbb{R}^{N}$.

Copyright $@$ by SIAM. Unauthorized reproduction of this article is prohibited. 
2.3. Lower estimates for nonnegative solutions in the case $m_{c}<m<1$. We recall the notation $m_{c}=(N-2 s)_{+} / N, \beta=1 /[2 s+N(m-1)]>0$ for $m>m_{c}$. The results we quote are valid for initial data in a weighted space $u_{0} \in L^{1}\left(\mathbb{R}^{N}, \varphi d x\right)$, where $\varphi$ satisfies the following conditions.

Assumption (A). The function $\varphi \in C^{2}\left(\mathbb{R}^{N}\right)$ is a positive real function that is radially symmetric and decreasing in $|x| \geq 1$. Moreover $\varphi$ satisfies

$$
0 \leq \varphi(x) \leq|x|^{-\alpha} \text { for }|x| \gg 1 \text { and } N-\frac{2 s}{1-m}<\alpha<N+\frac{2 s}{1-m} .
$$

We recall now Theorem 4.1 from [9] giving local lower bounds for the solution of the diffusion problem.

Theorem 2.4 (local lower bounds). Let $R_{0}>0, m_{c}<m<1$, and let $0 \leq$ $u_{0} \in L^{1}\left(\mathbb{R}^{N}, \varphi d x\right)$, where $\varphi$ is as in Assumption (A). Let $u(\cdot, t) \in L^{1}\left(\mathbb{R}^{N}, \varphi d x\right)$ be a very weak solution to the Cauchy problem (2.1), corresponding to the initial datum $u_{0}$. Then there exists a time

$$
t_{*}:=C_{*} R_{0}^{\frac{1}{\beta}}\left\|u_{0}\right\|_{L^{1}\left(B_{R_{0}}\right)}^{1-m}
$$

such that

$$
\inf _{x \in B_{R_{0} / 2}} u(x, t) \geq K_{1} R_{0}^{-\frac{2 s}{1-m}} t^{\frac{1}{1-m}} \text { if } 0 \leq t \leq t_{*}
$$

and

$$
\inf _{x \in B_{R_{0} / 2}} u(x, t) \geq K_{1} \frac{\left\|u_{0}\right\|_{L^{1}\left(B_{R_{0}}\right)}^{2 s \beta}}{t^{N \beta}} \text { if } t \geq t_{*} .
$$

The positive constants $C_{*}, K_{1}, K_{2}$ depend only on $m, s$, and $N \geq 1$.

The previous estimates, computed for $t=t_{*}$, are rewritten as

$$
\inf _{x \in B_{R_{0} / 2}} u(x, t) \geq K_{1} C_{*}^{\frac{1}{1-m}}\left\|u_{0}\right\|_{L^{1}\left(B_{R_{0}}\right)} R_{0}^{-N} .
$$

Then, if $R_{0}$ increases, the lower bound will decrease.

Concerning quantitative lower estimates, we recall Theorem 4.3 from [9].

Theorem 2.5 (global lower bounds when $m_{1}<m<1$ ). Under the conditions of Theorem 2.4 we have in the range $m_{1}<m<1$

$$
u(x, t) \geq \frac{C(t)}{|x|^{N+2 s}} \text { when }|x| \gg 1,
$$

valid for all $0<t<T$ with some bounded function $C>0$ that depends on $t, T$ and on the data.

TheOREM 2.6 (global lower bounds when $m_{c}<m<m_{1}$ ). Under the conditions of Theorem 2.4 we have in the range $m_{c}<m<m_{1}$

$$
u\left(x, t_{0}\right) \geq C(t)|x|^{-2 s /(1-m)}
$$

if $|x| \geq R$ and $0<t<t_{0}$.

The lower estimates for exponents $m>1$ need a new analysis that we supply in the next section.

Copyright $@$ by SIAM. Unauthorized reproduction of this article is prohibited. 
3. Lower parabolic estimate in the case $\boldsymbol{m}>\mathbf{1}$. We consider the FPME (with no reaction term) for $x \in \mathbb{R}^{N}$ and $t>0$ with nonnegative and integrable initial data

$$
u(x, 0)=u_{0}(x)
$$

and we also assume that $u_{0}$ is bounded and has compact support or decays rapidly as $|x| \rightarrow \infty$. We want to describe the behavior of the solution $u(x, t)>0$ as $|x| \rightarrow \infty$, more precisely, its rate of decay, for small times $t>0$. We take $m>1$ since the study of positivity for $m \leq 1$ was dealt with in previous results.

The first step in our asymptotic positivity analysis of solutions of (2.2) is to ensure that solutions with positive data remain positive and they have a precise tail behavior from below, which is based on a delicate subsolution construction.

THeOREM 3.1. Let $m>1$ and let $u(x, t)$ be a solution to (2.2) with initial data $u_{0}(x) \geq 0$ such that $u_{0}(x) \geq 1$ in the ball $B_{1}(0)$. Then there is a time $t_{1}>0$ and constants $C_{*}, R>0$ such that

$$
u(x, t) \geq C_{*} t|x|^{-(N+2 s)}
$$

if $|x| \geq R$ and $0<t<t_{1}$.

Proof. By comparison we may consider some smaller initial data $u_{0}$ such that $0 \leq u_{0}(x) \leq 1$ and $u_{0}(x)=1$ in the ball of radius 2 . Moreover, $u_{0}$ is smooth. By the results of [14] we know that $u(x, t) \in C^{\alpha}\left(\mathbb{R}^{N} \times[0, T]\right)$ and $u(x, t)>0$ for all $x \in \mathbb{R}^{N}$ and $t>0$. We have that $u(x, t) \geq 1 / 2$ in the ball of radius $1 / 2$ for all small times $0<t<t_{0}$.

- We want to construct a subsolution of the form

$$
U^{m}(x, t)=G(|x|)+t^{m} F^{m}(|x|) .
$$

We want to choose $G \geq 0$ and $F \geq 0$ in such a way that $U$ will be a formal subsolution of the FPME in a domain of the form $Q=\left\{|x| \geq 1 / 2,0<t<t_{1}\right\}$, i.e., we want $U_{t}+(-\Delta)^{s} U^{m} \leq 0$ in $Q$. Note that

$$
U_{t}=\left(G(|x|)+t^{m} F^{m}(|x|)\right)^{(1 / m)-1} t^{m-1} F^{m}(|x|) \leq F(|x|) .
$$

We also have, with $L_{s}=(-\Delta)^{s}$,

$$
L_{s} U^{m}=L_{s} G(|x|)+t^{m} L_{s} F^{m}(|x|) .
$$

We take $F$ positive, smooth and $F(r) \sim r^{-(N+2 s)}$ as $r \rightarrow \infty$ to get the desired conclusion after the comparison argument: $u(x, t) \geq U(x, t) \geq c t r^{-(N+2 s)}$ if $r$ is large and $t \sim 0$. For later use, let us say that $F \leq C_{2} r^{-(N+2 s)}$ for $r>1 / 2$. Since $m>1$ we can choose $F$ smooth so that $L_{s} F^{m}=O\left(r^{-(N+2 s)}\right)$ for $r>1 / 2$ (use the asymptotic estimates like the first lemma in [9]).

We will take $G(r)=0$ for $r=|x| \geq 1 / 2$ so that $U(x, t)=t F(|x|)$ there. If $G$ is also smooth we have $L_{s} G$ bounded and $L_{s} G \sim-C_{1} r^{-(N+2 s)}$ as $r \rightarrow \infty$. By contracting $G$ in space, $\widetilde{G}(x)=G(k x), k>0$, we may then say that $L_{s} G \leq-C_{1} r^{-(N+2 s)}$ for $r>1 / 2$. Then we will have for $r>1 / 2$ and $0<t \ll 1$ that

$$
\begin{aligned}
U_{t}+L_{s} U^{m} & \leq F+L_{s} G+t^{m} L_{s} F^{m} \leq C_{2} r^{-(N+2 s)}-C_{1} r^{-(N+2 s)}+t^{m} L_{s} F^{m} \\
& \leq\left(C_{2}+\varepsilon\right) r^{-(N+2 s)}-C_{1} r^{-(N+2 s)} \leq 0
\end{aligned}
$$

if $C_{1}>C_{2}$. We can choose $G$ large so that $C_{1}$ is large enough. 
- We now want to use the viscosity method to compare $u(x, t)$ with $U(x, t)$ in the region $Q=\left\{|x| \geq 1 / 2,0<t<t_{1}\right\}$, and this will prove that $U(x, t) \leq u(x, t)$ in $Q$. Apart from the subsolution condition that we have checked, we need a suitable comparison of the boundary conditions at $r=1 / 2$,

$$
U(1 / 2, t)=t F(1 / 2) \leq 1 / 2 .
$$

This ends the construction if the comparison result is justified. The contradiction argument at the first point of contact between $u$ and $U$ will be justified as in [9] (where it was applied to fast diffusion equations of fractional diffusion type) if the solution we have is a bit smooth: $u_{t}$ and $L_{s} u^{m}$ must be continuous and the equation must be satisfied pointwise there. This regularity is true and the proofs are under study now.

Alternatively, we may use implicit time discretization with a sequence of approximations. The justification of the method in the elliptic case is done in the paper [28] on symmetrization techniques.

Remark. The level $u_{0}(x) \geq 1$ in the ball $B_{1}(0)$ can be replaced by $u_{0}(x) \geq \varepsilon>0$ in any other ball by means of translation and scaling. In this way the result is true for all continuous and nonnegative initial data $u_{0}$ and is of course nontrivial.

4. Evolution of level sets of solutions to problem (1.1). In this section we start the proof of the main result of the paper on evolution of level sets with exponential speed of propagation. In a first step we prove the convergence to zero on outer sets. Since the decay assumption on the initial data is the same for $m_{1}<m<1$ and $m>1$, we will treat both cases, as well as $m=1$, in the following lemma.

LEMma 4.1. We consider $m>m_{1}$ and let $u$ be the solution of problem (1.1) with initial datum $u_{0}(x) \in L^{1}\left(\mathbb{R}^{N}\right), 0 \leq u_{0} \leq 1$. We assume that $u_{0}$ satisfies the decay property

$$
u_{0}(x) \leq C|x|^{-(N+2 s)} \quad \forall x \in \mathbb{R}^{N} .
$$

Then, for $\sigma>\sigma_{3}$ if $m>1$ (respectively, for $\sigma>\sigma_{2}$ if $m_{1}<m \leq 1$ ), we have

$$
u(x, t) \rightarrow 0 \quad \text { as } t \rightarrow \infty
$$

uniformly for $|x| \geq e^{\sigma t}$.

Proof. We consider the solution $\bar{u}(x, t)$ of the linearized problem

$$
\bar{u}_{t}+L_{s} \bar{u}^{m}=f^{\prime}(0) \bar{u}, \quad \bar{u}(0, x)=u_{0}(x) .
$$

Since $f$ is a concave function, we have $f^{\prime}(0) s \geq f(s)$ for all $s \in[0,1]$, and thus $\bar{u}$ is a supersolution of problem (1.1), which implies the upper estimate

$$
u(x, t) \leq \bar{u}(x, t) \quad \text { for } t \geq 0, x \in \mathbb{R}^{N} .
$$

Next, we define $\tilde{v}(x, \tau)$ by

$$
\tilde{v}(x, \tau)=e^{-f^{\prime}(0) t} \bar{u}(x, t)
$$

and new time

$$
\begin{aligned}
\tau & =\frac{1}{(m-1) f^{\prime}(0)}\left[e^{(m-1) f^{\prime}(0) t}-1\right] \text { if } m>1, \\
\tau & =\frac{1}{(1-m) f^{\prime}(0)}\left[1-e^{-(1-m) f^{\prime}(0) t}\right] \text { if } m<1,
\end{aligned}
$$

Copyright $@$ ( ) by SIAM. Unauthorized reproduction of this article is prohibited. 
and $\tau=t$ for $m=1$. It is immediate to check that $\tilde{v}(x, \tau)$ is a solution of the FPME (1.10) with initial datum $\tilde{v}_{0}=u_{0}$. Let $B_{M}(x, \tau)$ the Barenblatt solution with mass $M$ of the FPME, as defined in section 2.2. By virtue of the properties of the Barenblatt solutions and assumption (4.1) on the initial data, we conclude there exists $M>0$ big enough and $\tau_{0}>0$ such that

$$
\tilde{v}_{0}(x) \leq B_{M}\left(x, \tau_{0}\right)
$$

By the maximum principle

$$
\tilde{v}(x, \tau) \leq B_{M}\left(x, \tau+\tau_{0}\right) \quad \forall x \in \mathbb{R}^{N}, t>0 .
$$

Now, using the characterization of the decay of the Barenblatt profile given by relation (2.5), we obtain that there exists $K_{1}>0$ such that $F_{M}(r) \leq K_{1} r^{-(N+2 s)}$ for all $r \geq 0$. We obtain the following upper estimate on the solution $u$ of problem (1.1):

$$
\begin{aligned}
u(x, t) & \leq \bar{u}(x, t)=e^{f^{\prime}(0) t} \tilde{v}(x, \tau) \\
& \leq e^{f^{\prime}(0) t} B_{M}\left(x, \tau+\tau_{0}\right)=e^{f^{\prime}(0) t}\left(\tau+\tau_{0}\right)^{-\alpha} F_{M}\left(|x|\left(\tau+\tau_{0}\right)^{-\beta}\right) \\
& \left.\leq e^{f^{\prime}(0) t}\left(\tau+\tau_{0}\right)^{-\alpha} K_{1}\left(|x|\left(\tau+\tau_{0}\right)^{-\beta}\right)\right)^{-(N+2 s)} \\
& =K_{1} e^{f^{\prime}(0) t}\left(\tau+\tau_{0}\right)^{2 \beta s}|x|^{-(N+2 s)} .
\end{aligned}
$$

Case $m>1$. In order to continue the estimate, we remark that for large times $t$, the term $\tau^{2 \beta s}$ has an influence on the result only in the case $m>1$. Then $\left(\tau+\tau_{0}\right)^{2 \beta s} \leq$ $e^{(m-1) f^{\prime}(0) t}$ for large $t$. Let us assume that $|x| \geq e^{\sigma t}$. Then one has

$$
u(x, t) \leq C K_{1} e^{f^{\prime}(0) t} \tau^{2 \beta s} e^{-\sigma(N+2 s) t}=C K_{1} e^{\left[f^{\prime}(0)+2 f^{\prime}(0)(m-1) \beta s-\sigma(N+2 s)\right] t} .
$$

We want to have $f^{\prime}(0)+2 f^{\prime}(0)(m-1) \beta s-\sigma(N+2 s)<0$, which is just the condition

$$
\sigma>\frac{1+2(m-1) \beta s}{N+2 s} f^{\prime}(0)=\sigma_{3} .
$$

We have obtained the convergence of $u(x, t)$ to 0 as $t \rightarrow \infty$ for $|x| \geq e^{\sigma t}$.

Case $m \leq 1$. In this case, the term $\left(\tau+\tau_{0}\right)^{2 \beta s}$ is bounded for every $t>0$, as we can see from (4.5). As before, we assume $|x| \geq e^{\sigma t}$. Then, we get

$$
u(x, t) \leq C K_{1} e^{f^{\prime}(0) t} e^{-\sigma(N+2 s) t}=C K_{1} e^{\left[f^{\prime}(0)-\sigma(N+2 s)\right] t} .
$$

For $\sigma>\sigma_{2}=\frac{f^{\prime}(0)}{N+2 s}$, the exponent is negative $f^{\prime}(0)-\sigma(N+2 s)<0$ and we obtain the convergence of $u(x, t)$ to 0 as $t \rightarrow \infty$.

LEMMA 4.2. We consider $m_{c}<m<m_{1}$. Let $u$ be the solution of problem (1.1) with initial datum $u_{0}(x) \in L^{1}\left(\mathbb{R}^{N}\right), 0 \leq u_{0} \leq 1$, and we assume $u_{0}$ satisfies the decay property

$$
u_{0}(x) \leq C|x|^{-2 s /(1-m)} \quad \forall x \in \mathbb{R}^{N} .
$$

Then, for $\sigma>\sigma_{1}$ we have

$$
u(x, t) \rightarrow 0, \quad t \rightarrow \infty
$$

uniformly for $|x| \geq e^{\sigma t}$. 
Proof. The proof follows that of Lemma 4.1 since the Barenblatt solution $B_{M}(x, \tau)=$ $\tau^{-\alpha} F_{M}\left(|x| \tau^{-\beta}\right)$ of the diffusion problem satisfies $F_{M}(r) \sim r^{-2 s /(1-m)}$ according to Theorem 2.2. Therefore, we obtain the estimate

$$
\begin{aligned}
u(x, t) & \left.\leq e^{f^{\prime}(0) t}\left(\tau+\tau_{0}\right)^{-\alpha} K_{1}\left(|x|\left(\tau+\tau_{0}\right)^{-\beta}\right)\right)^{-2 s /(1-m)} \\
& =K_{1} e^{f^{\prime}(0) t}\left(\tau+\tau_{0}\right)^{1 /(1-m)}|x|^{-2 s /(1-m)} .
\end{aligned}
$$

Since $m<1$, the term $\left(\tau+\tau_{0}\right)^{1 /(1-m)}$ is controlled by $e^{f^{\prime}(0) t}$ and then for $|x| \geq e^{\sigma t}$ we obtain

$$
u(x, t) \leq K_{1} e^{f^{\prime}(0) t-2 s \sigma t /(1-m)} .
$$

For $\sigma>\frac{1-m}{2 s} f^{\prime}(0)=\sigma_{1}$ we obtain the desired convergence to 0 as $t \rightarrow \infty$.

Remarks.

I. When $m=1$ we recover the minimal speed $\sigma_{2}=f^{\prime}(0) /(N+2 s)$ obtained by Cabré and Roquejoffre in [12]. The proof is similar, but in the nonlinear case we have to make an exponential change of time variable. Note also that we only use the decay properties of the fundamental solution.

II. The value of the critical exponent $\sigma_{2}$ can be easily obtained from the following formal study of the level lines of $u(x, t)$. Thus, the set $\{u(x, t) \sim \epsilon\}$ can be written in terms of $\tilde{v}(x, \tau)$ defined in (4.3) as

$$
e^{f^{\prime}(0) t} \tilde{v}(x, \tau) \sim \epsilon .
$$

By Theorem 2.3, $\tilde{v}(x, \tau)$ behaves like the Barenblatt solution of the FPME (2.1) (we discuss only the case $m>m_{1}$ ):

$$
\tilde{v}(x, \tau) \sim B(x, \tau)=\tau^{-\alpha} F(r), \quad F(r) \sim r^{-(N+2 s)}, \quad r=|x| \tau^{-\beta} .
$$

From [26], we know that $B(x, \tau) \sim \tau^{-\alpha+\beta(N+2 s)}|x|^{-(N+2 s)}$, thus $\tilde{v}(x, \tau) \sim$ $\tau^{2 \beta s}|x|^{-(N+2 s)}$. At this moment, (4.6) implies $e^{f^{\prime}(0) t} \tau^{2 \beta s}|x|^{-(N+2 s)} \sim \epsilon$.

For instance, in the $m>1$ case, it follows that

$$
|x| \sim\left(\frac{1}{\epsilon} e^{(1+2 \beta s(m-1)) f^{\prime}(0) t}\right)^{1 /(N+2 s)} \sim e^{\frac{1+2 \beta s(m-1)}{N+2 s} f^{\prime}(0) t},
$$

and we deduce an exponential behavior of the level sets $|x| \sim e^{\sigma_{3} t}$, where $\sigma_{3}=\frac{1+2 \beta s(m-1)}{N+2 s} f^{\prime}(0)$. Similarly, in the $m_{1}<m<1$ case, we get that

$$
|x| \sim\left(\frac{1}{\epsilon} e^{f^{\prime}(0) t}\right)^{1 /(N+2 s)} \sim e^{\sigma_{2} t}, \quad \sigma_{2}=\frac{f^{\prime}(0)}{N+2 s} .
$$

5. Evolution of level sets II. Convergence to 1 on inner sets. In this section, we will prove the convergence to 1 of the solution $u(x, t)$ of problem (1.1), i.e., the second part of the statements of our main theorems, Theorems 1.1, 1.2, and 1.3.

5.1. Case $\boldsymbol{m}>\boldsymbol{m}_{\mathbf{1}}$. We will present this case in full detail. The proof for the case $m_{c}<m<m_{1}$ being similar, we will sketch it at the end of this section. We have $N \geq 1, s \in(0,1), m>m_{1}, f$ satisfies (1.2), and $\sigma_{2}=\frac{f^{\prime}(0)}{N+2 s}$ as defined in (1.6).

Proposition 5.1. Let $N \geq 1, s \in(0,1), m_{1}<m$, $f$ satisfying (1.2). Let $u$ be a solution of problem (1.1) with initial datum $0 \leq u_{0}(\cdot) \leq 1, u_{0} \neq 0$. Then for every $\sigma \in\left(0, \sigma_{2}\right), u(x, t) \rightarrow 1$ uniformly on $\left\{|x| \leq e^{\sigma t}\right\}$ as $t \rightarrow \infty$. 
Proof. We fix $\sigma \in\left(0, \sigma_{2}\right)$. Proving the convergence of $u(x, t)$ to 1 is equivalent to proving the convergence of $1-u^{m}$ to 0 . Therefore, we fix $\lambda>0$ and we need to find a time $t_{\lambda}$ large enough such that $1-u^{m}(x, t) \leq \lambda$ for all $t \geq t_{\lambda}$ and $|x| \leq e^{\sigma t}$.

- Let us accept for the moment the following lower estimate that will be proved later as Lemma 5.5: given $\nu \in\left(\sigma, \sigma_{2}\right)$, there exist $\epsilon \in(0,1)$ and $t_{0}>0$ such that

$$
u \geq \epsilon \quad \text { for } t \geq t_{0} \text { and }|x| \leq e^{\nu t} .
$$

We now proceed with the last part of the argument, where the effect of the nonlinear diffusion is most clearly noticed. We take $t_{1} \geq t_{0}$ and consider the inner sets where

$$
\epsilon \leq u \leq 1 \quad \text { for }(x, t) \in \Omega_{I}:=\left\{t \geq t_{1},|x| \leq e^{\nu t}\right\} .
$$

Let $v=1-u^{m}$. Then $v$ satisfies the equation

$$
\frac{1}{m}(1-v)^{\frac{1}{m}-1} v_{t}+L_{s} v+f(u)=0,
$$

which we write in the form

$$
a(x, t) v_{t}+L_{s} v+b(x, t) v=0, \quad a(x, t)=\frac{1}{m} u^{1-m}, \quad b(x, t)=\frac{f(u)}{v} .
$$

Moreover, we estimate $a(x, t)$ as

$$
a_{0}=\frac{1}{m} \epsilon^{1-m} \leq a(x, t) \leq a_{1}:=\frac{1}{m} \text { in } \Omega_{I} \text { if } m<1,
$$

respectively,

$$
a_{0}=\frac{1}{m} \leq a(x, t) \leq a_{1}:=\frac{1}{m} \epsilon^{1-m} \text { in } \Omega_{I} \text { if } m>1 .
$$

We argue similarly for $b(x, t)$ in $\Omega_{I}$ :

$$
b(x, t)=\frac{f(u)}{1-u^{m}}=\frac{f(u)}{(1-u) m \xi^{m-1}} \geq b_{0}, \quad \xi \in(u, 1),
$$

where

$$
b_{0}=\frac{1}{m} \frac{f(\epsilon)}{1-\epsilon} \epsilon^{1-m} \text { if } m<1 \quad \text { and } \quad b_{0}=\frac{1}{m} \frac{f(\epsilon)}{1-\epsilon} \text { if } m>1 .
$$

In particular, $v$ satisfies

$$
a(x, t) v_{t}+L_{s} v+b_{0} v \leq 0 \quad \text { in } \Omega_{I} .
$$

- We look for a supersolution $w$ to problem (5.3) that will be found as a solution to a linear problem with constant coefficients, and we also need that $w_{t} \leq 0$. More precisely, we consider $w$ solution of the concrete problem

$$
\begin{cases}a_{1} w_{t}(x, t)+L_{s} w(x, t)+b_{0} w=0 & \text { for } x \in \mathbb{R}^{N} \text { and } t>t_{1}, \\ w\left(x, t_{1}\right)=1+\frac{1}{C_{2}}|x|^{\gamma} & \text { for } x \in \mathbb{R}^{N},\end{cases}
$$

where the exponent $\gamma$ taken such that

$$
0<\gamma:=\frac{1}{\nu} \frac{b_{0}}{a_{1}}<2 s .
$$

Copyright $@$ by SIAM. Unauthorized reproduction of this article is prohibited. 
We can eventually consider a smaller $\epsilon$ for this inequality to hold. Equation (5.5) is linear, and the solution can be computed explicitly,

$$
w(x, t)=e^{-\frac{b_{0}}{a_{1}}\left(t-t_{1}\right)} \bar{w}(x, \tau), \quad \tau=\frac{1}{a_{1}}\left(t-t_{1}\right),
$$

where $\bar{w}(x, \tau)$ solves the linear problem

$$
\bar{w}_{\tau}(x, \tau)+L_{s} \bar{w}(x, \tau)=0, \quad \bar{w}(0)=1+\frac{1}{C_{2}}|x|^{\gamma} .
$$

We observe that $\tilde{w}$ can be written in the form

$$
\bar{w}(x, \tau)=1+\frac{1}{C_{2}} U\left(x, \tau+\theta_{1}\right)
$$

where

$$
U(x, \tau)=\tau^{\alpha_{1}} F\left(|x| \tau^{-\beta_{1}}\right), \quad \alpha_{1}=\frac{\gamma}{2 s}, \beta_{1}=\frac{1}{2 s},
$$

is the self-similar solution of the linear problem

$$
U_{\tau}(x, \tau)+L_{s} U(x, \tau)=0, \quad U(x, 0)=|x|^{\gamma} .
$$

The properties of the self-similar solutions $U(x, \tau)$ deserve a separate study, which is done in detail in section 6 . Thus, by Lemma 6.3 the profile $F$ is nondecreasing and $U(x, \tau)$ has a spatial decay as $|x|^{\gamma}$ for large $|x| \tau^{-1 / 2 s}$ :

$$
C_{2}|x|^{\gamma} \leq U(x, \tau) \leq C_{1}|x|^{\gamma} \quad \forall \quad|x| \tau^{-1 / 2 s} \geq K_{1} .
$$

We will consider a suitable delay time $\tau_{1}$ in the definition of $\bar{w}$ stated in (5.7). In what follows we will use the notation $\eta=|x| \tau^{-\beta_{1}}$. We check that the derivative $w_{t}$ is negative:

$$
\begin{aligned}
w_{t}(x, t)= & \frac{d}{d t}\left[e^{-\frac{b_{0}}{a_{1}}\left(t-t_{1}\right)}\left(1+C_{2}^{-1} U\left(x, \tau+\tau_{1}\right)\right)\right] \\
= & e^{-\frac{b_{0}}{a_{1}}\left(t-t_{1}\right)}\left[-\frac{b_{0}}{a_{1}}\left(1+C_{2}^{-1}\left(\tau+\tau_{1}\right)^{\alpha_{1}} F(\eta)\right)\right. \\
& \left.+C_{2}^{-1}\left(\tau+\tau_{1}\right)^{\alpha_{1}-1}\left(\alpha_{1} F(\eta)-\beta_{1} \eta F^{\prime}(\eta)\right) \frac{d \tau}{d t}\right] \\
= & e^{-\frac{b_{0}}{a_{1}}\left(t-t_{1}\right)} \frac{1}{a_{1} C_{2}}\left[-b_{0} C_{2}+\left(-b_{0}\left(\tau+\tau_{1}\right)+\alpha_{1}\right)\left(\tau+\tau_{1}\right)^{\alpha_{1}-1} F(\eta)\right. \\
& \left.-\beta_{1}\left(\tau+\tau_{1}\right)^{\alpha_{1}-1} \eta F^{\prime}(\eta)\right] .
\end{aligned}
$$

Since $F^{\prime}(\eta)>0$ for all $\eta>0$, we get that $w_{t}(x, t) \leq 0$ for all $t \geq t_{1}$ if $\tau+\tau_{1} \geq \alpha_{1} / b_{0}$, which is true for a suitable choice of $\tau_{1}$.

- Now we can compare $w$ and $v$ by applying the maximum principle stated in Lemma 7.1 of the appendix, as in [12]. Define $\bar{v}=v-w$ and ensure the hypothesis of the lemma are satisfied.

(H1) We check that $w\left(x, t_{1}\right) \geq v\left(x, t_{1}\right)$ for all $x \in \mathbb{R}^{N}$ :

$$
w\left(x, t_{1}\right) \geq 1>v=1-u^{m} \quad \forall x \in \mathbb{R}^{N} .
$$

Copyright $@$ ( ) by SIAM. Unauthorized reproduction of this article is prohibited. 
(H2) We check that $w \geq v$ in $\left(\left(t_{1}, \infty\right) \times \mathbb{R}^{N}\right) \backslash \Omega_{I}$, that is, $t \geq t_{1}$ and $|x| \geq e^{\nu t}$. At this point, we use the estimates (5.8). We ensure that $e^{\nu t} \geq K_{1}\left(\tau+\tau_{1}\right)^{1 / 2 s}$ for all $t \geq t_{1}$, which is true by choosing eventually a larger $t_{1}$. Therefore

$$
\begin{aligned}
w(x, t) & =e^{-\frac{b_{0}}{a_{1}}\left(t-t_{1}\right)} \bar{w}(\tau, x) \geq e^{-\frac{b_{0}}{a_{1}}\left(t-t_{1}\right)}\left(1+\frac{1}{C_{2}} C_{2}|x|^{\gamma}\right) \\
& \geq e^{-\frac{b_{0}}{a_{1}}\left(t-t_{1}\right)}\left(1+e^{\gamma \nu t}\right) \geq 1 \geq v(x, t) \quad \forall t \geq t_{1},|x| \geq e^{\nu t}
\end{aligned}
$$

since $\gamma$ satisfies (5.6). By the previous computation $\bar{v} \leq 0$ in $\left(\left(t_{1}, \infty\right) \times \mathbb{R}^{N}\right) \backslash \Omega_{I}$.

(H3) The next step is to prove that $\bar{v}$ is a subsolution of problem (5.4). Indeed, we have that

$$
\begin{aligned}
a(x, t) \bar{v}_{t}+L_{s} \bar{v}+b_{0} \bar{v}= & a(x, t) v_{t}+L_{s} v+b_{0} v-\left[a_{1} w_{t}+L_{s} w+b_{0} w\right] \\
& +\left(a_{1}-a(x, t)\right) w_{t} \leq 0
\end{aligned}
$$

in $\Omega_{I}$. By Lemma 7.1 we obtain that $\bar{v} \leq 0$ in $\left[t_{1}, \infty\right) \times \mathbb{R}^{N}$ for $t_{1}$ taken to be large enough. Thus,

$$
v(x, t) \leq w(x, t)=e^{-\frac{b_{0}}{a_{1}}\left(t-t_{1}\right)}\left(1+C_{2}^{-1} U\left(x, \tau+\tau_{1}\right)\right) \leq e^{-\frac{b_{0}}{a_{1}}\left(t-t_{1}\right)}\left(1+\frac{C_{1}}{C_{2}}|x|^{\gamma}\right) .
$$

- Let us consider the inner set $(x, t) \in\left\{t \geq t_{\lambda},|x| \leq C_{\lambda} e^{\nu t}\right\}$. We have

$$
v(x, t) \leq e^{-\frac{b_{0}}{a_{1}}\left(t-t_{1}\right)}\left(1+\frac{C_{1}}{C_{2}} C_{\lambda}^{\gamma} e^{\gamma \nu t}\right) \leq e^{-\frac{b_{0}}{a_{1}}\left(t_{\lambda}-t_{1}\right)}+\frac{C_{1}}{C_{2}} e^{\frac{b_{0}}{a_{1}} t_{1}} C_{\lambda}^{\gamma} \leq \lambda
$$

for $C_{\lambda}$ small enough and $t_{\lambda}$ large enough.

Finally, since $\sigma<\nu$, then $e^{\sigma t} \leq C_{\lambda} e^{\nu t}$ for every $t \geq t_{\lambda}$ with $t_{\lambda}$ large enough, and the previous inequality implies that

$$
1-u^{m}(x, t)=v(x, t) \leq \lambda \quad \text { for } \quad t \geq t_{\lambda},|x| \leq e^{\sigma t},
$$

which concludes the proof of the uniform convergence to the level $u=1$.

To complete the proof of the result of this subsection, we need to supply the proof of the lower estimate (5.1). This will be done in three steps.

Step I. Starting with arbitrary initial datum $0 \leq u_{0} \leq 1, u_{0} \neq 0$, we obtain a lower bound for $u$ with the desired tail $u \geq c|x|^{-(\bar{N}+2 s)}$ for large $|x|$. The result corresponds to Lemma 5.2.

Step II. We prove that given an initial data taking the value $\epsilon$ in the ball of radius $\rho_{0}$ and decaying like that $|x|^{-(N+2 s)}$ for large $|x|$, the corresponding solution of problem (1.1) will be raised to at least the same level $\epsilon$ in a larger ball $\rho_{1}$ and in a later time that is estimated. The sizes are important. This will be Lemma 5.3.

Step III. By combining the previous two results, we conclude that $u \geq \epsilon$ on the inner sets for a certain $\epsilon>0$. This will be Lemmas 5.4 and 5.5.

Steps II and III follow the ideas of [12] in the linear case, with a long technical adaptation to nonlinear diffusion.

Lemma 5.2 (long tail behavior). Let $N \geq 1, s \in(0,1), m>m_{1}$, $f$ satisfying (1.2) and $\sigma \in\left(0, \sigma_{2}\right)$. Let $u$ be the solution of problem (1.1) with initial datum $u(0, \cdot)=u_{0}$, where $0 \leq u_{0} \leq 1, u_{0} \neq 0$. Then for any fixed $t_{0}>0$ there exist $\epsilon \in(0,1), a_{0}>0, \rho_{0}>1$ such that

$$
u(x, t) \geq v_{0}(x):= \begin{cases}a_{0}|x|^{-(N+2 s)}, & |x| \geq \rho_{0}, \\ \epsilon=a_{0} \rho_{0}^{-(N+2 s)}, & |x| \leq \rho_{0},\end{cases}
$$

for all $t \in\left[t_{0}, 2 t_{0}\right], x \in \mathbb{R}^{N}$. 
Proof. We recall that $\sigma_{2}=f^{\prime}(0) /(N+2 s)$. The idea is to view $u(x, t)$ the solution of problem (1.1) as a supersolution of the homogeneous problem with the same initial datum $u_{0}$, that is, the FPME. Therefore,

$$
u\left(x, t_{0}+t\right) \geq \underline{u}(x, t) \quad \forall t \geq 0, \quad x \in \mathbb{R}^{N},
$$

where $\underline{u}$ is the solution of the FPME with initial datum $u_{0}$

$$
\begin{cases}\underline{u}_{t}(x, t)+L_{s} \underline{u}^{m}(x, t)=0 & \text { for } x \in \mathbb{R}^{N} \text { and } t>0, \\ \underline{u}(x, 0)=u_{0}(x) & \text { for } x \in \mathbb{R}^{N} .\end{cases}
$$

We will estimate $\underline{u}$ from below by using the local and global estimates on the FPME given in Theorems 2.4 and 2.5 for $m<1$, respectively, Theorem 3.1 for $m>1$. The decay in case $m=1$ is well known; see section 6 for a review. More exactly, in all cases $m>m_{1}$, there exist a time $T>0$ and constant $R>0$ such that

$$
u(x, t) \geq C(t)|x|^{-(N+2 s)} \quad \forall|x| \geq R, 0<t<T .
$$

Then, for a fixed $t_{*} \in(0, T)$ which also satisfies $t_{*}<t_{0}$, we can find a Barenblatt solution $B_{M}(x, t)$ and a time $t_{2}>0$ such that

$$
u\left(x, t_{*}\right) \geq B_{M}\left(x, t_{2}\right) \quad \forall x \in \mathbb{R}^{N},
$$

and therefore, by the comparison principle

$$
u\left(x, t+t_{*}\right) \geq B_{M}\left(x, t+t_{2}\right) \quad \forall x \in \mathbb{R}^{N}, t \geq 0 .
$$

In particular, we can choose $\epsilon>0$ such that

$$
u(x, t) \geq v_{0}(x):= \begin{cases}a_{0}|x|^{-(N+2 s)}, & |x| \geq \rho_{0}, \\ \epsilon=a_{0} \rho_{0}^{-(N+2 s)}, & |x| \leq \rho_{0},\end{cases}
$$

for all $x \in \mathbb{R}^{N}, t \in\left[t_{0}, 2 t_{0}\right]$.

Lemma 5.3 (positivity for a sequence of times). Let $m>m_{1}$. For every $\sigma<\sigma_{2}$ there exist $t_{0} \geq 1$ and $0<\epsilon_{0}<1$ depending only on $N, s, f$, and $\sigma$ for which the following holds: given $\rho_{0} \geq 1$ and $0<\epsilon \leq \epsilon_{0}$, letting $a_{0}>0$ be defined by $a_{0} \rho_{0}^{-(N+2 s)}=\epsilon$, if we take

$$
v_{0}(x)= \begin{cases}a_{0}|x|^{-(N+2 s)}, & |x| \geq \rho_{0}, \\ \epsilon=a_{0} \rho_{0}^{-(N+2 s)}, & |x| \leq \rho_{0},\end{cases}
$$

then the solution $v$ of problem (1.1) with initial condition $v_{0}$ satisfies

$$
v\left(x, k t_{0}\right) \geq \epsilon \quad \text { for }|x| \leq \rho_{0} e^{\sigma k t_{0}}
$$

for all $k \in\{0,1,2,3, \ldots\}$.

Proof of Lemma 5.3 in the case $m>1$. I. Preliminary choices. From the beginning we fix $\sigma \in\left(0, \sigma_{2}\right)$. We take $\delta \in(0,1)$ small enough such that

$$
\frac{f(\delta)}{(N+2 s) \delta} \geq \sigma, \quad \frac{f(\delta)}{(N+2 s) \delta} \geq N(m-1) \beta \sigma_{2} .
$$

Copyright (C) by SIAM. Unauthorized reproduction of this article is prohibited. 
For example, take $\delta$ such that

$$
\frac{f(\delta)}{(N+2 s) \delta}=\frac{1}{2}\left(\sigma_{2}+\max \left\{\sigma, N(m-1) \beta \sigma_{2}\right\}\right) .
$$

This choice will be explained later. Next we take $t_{0}$ sufficiently large depending only on $N, s, u_{0}$, and $\sigma$ such that

$$
e^{t_{0} 2 \beta s \frac{f(\delta)}{\delta}} \geq\left(1+t_{0} / c_{2}\right)^{N \beta} K_{3}, \quad\left(K_{2} / 2 K_{1}\right)^{1 /(N+2 s)} e^{\frac{f(\delta)}{(N+2 s) \delta} t_{0}} \geq e^{\sigma t_{0}},
$$

where $K_{2}<2 K_{1}$ are constants describing the properties of the profile $F_{1}$ of the Barenblatt function with mass 1 given in (2.9) and (2.10), and we recall for convenience that

$$
K_{2}\left(1+r^{N+2 s}\right)^{-1} \leq F_{1}(r) \leq K_{1} r^{-(N+2 s)} \quad \forall r>0 .
$$

Throughout the proof there will appear several expressions involving the three parameters $K_{1}, K_{2}$, and $F_{1}(0)$. We introduce here the notation used:

$$
c_{1}=K_{1}^{-\frac{N}{N+2 s}} F_{1}(0)^{-\frac{2 s}{N+2 s}}, \quad c_{2}=K_{1}^{-\frac{2 s}{N+2 s}} F_{1}(0)^{m-1+\frac{2 s}{N+2 s}}, \quad K_{3}=2 F_{1}(0) K_{2}^{-1} .
$$

Define now $\epsilon_{0}$ by

$$
\epsilon_{0}=\delta e^{-(f(\delta) / \delta) t_{0}}
$$

Now, we fix $0<\epsilon<\epsilon_{0}$ and $\rho_{0}>1$.

II. First step of the iteration $k=1$. We will do a very detailed analysis of the case $k=1$, which is then iterated for the rest of values of $k$.

IIa. Construction of subsolutions to problem (1.1). Let $w$ be a solution of the problem with linearized reaction

$$
\begin{cases}w_{t}(x, t)+L_{s} w^{m}(x, t)=\frac{f(\delta)}{\delta} w & \text { for } x \in \mathbb{R}^{N} \text { and } t>0, \\ w(0, x)=v_{0}(x) & \text { for } x \in \mathbb{R}^{N}\end{cases}
$$

We define $\bar{w}(x, \tau)$ by

$$
\bar{w}(x, \tau)=e^{-\frac{f(\delta)}{\delta} t} w(x, t)
$$

with a new time

$$
\tau=\frac{1}{(m-1) f(\delta) / \delta}\left[e^{(m-1) \frac{f(\delta)}{\delta} t}-1\right] \text { if } m>1,
$$

so that $\tau=t$ in the limit $m=1$. Then, $\bar{w}$ is a solution of the FPME with initial datum $v_{0}$

$$
\begin{cases}\bar{w}_{\tau}(x, \tau)+L_{s} \bar{w}^{m}(x, \tau)=0 & \text { for } x \in \mathbb{R}^{N} \text { and } \tau>0 \\ \bar{w}(x, 0)=v_{0}(x) & \text { for } x \in \mathbb{R}^{N}\end{cases}
$$

IIb. Comparison with a Barenblatt solution. Lower bound for $v\left(x, t_{0}\right)$. We prove that there exist $M_{1}>0$ and $\theta_{1}>0$ such that

$$
v_{0}(x) \geq B_{M_{1}}\left(x, \theta_{1}\right) \quad \forall x \in \mathbb{R}^{N},
$$

Copyright $@$ by SIAM. Unauthorized reproduction of this article is prohibited. 
where $B_{M_{1}}(x, \tau)$ is the Barenblatt solution of problem (1.10) with mass $M_{1}$ given by Theorem 2.1:

$$
B_{M_{1}}(x, \tau)=M_{1} B_{1}\left(x, M_{1}^{m-1} \tau\right) .
$$

Now, $B_{M_{1}}(x, \tau)$ can be written in terms of the profile $F_{1}$ as

$$
B_{M_{1}}(x, \tau)=M_{1}^{1-(m-1) \alpha} \tau^{-\alpha} F_{1}\left(\left(M_{1}^{m-1} \tau\right)^{-\beta}|x|\right) .
$$

We will use the properties of the profile $F_{1}$ stated in (2.9) and (2.10). With this information, we will find the constants $M_{1}>0$ and $\theta_{1}>0$ such that inequality (5.19) at the initial time holds true. For $|x| \leq \rho_{0}$ we have that $B_{M_{1}}\left(x, \theta_{1}\right) \leq M_{1}^{1-(m-1) \alpha} \theta_{1}^{-\alpha} F_{1}(0)$. Note that $1-(m-1) \alpha=2 \beta s>0$. We impose the first condition

$$
M_{1}^{2 \beta s} \theta_{1}^{-\alpha} F_{1}(0) \leq \epsilon
$$

Next we look at the tail $|x| \geq \rho_{0}$. Since we have

$$
B_{M_{1}}\left(x, \theta_{1}\right) \leq M_{1}^{2 \beta s} \theta_{1}^{-\alpha} K_{1}\left(\left(M_{1}^{m-1} \theta_{1}\right)^{-\beta}|x|\right)^{-(N+2 s)}
$$

in order to use this inequality for large $|x|$ we also impose the condition

$$
K_{1} M_{1}^{1+2 \beta s(m-1)} \theta_{1}^{2 \beta s} \leq a_{0}, \quad \text { where } a_{0}=\epsilon \rho_{0}^{N+2 s} .
$$

Conditions (5.22) and (5.23) are sufficient for inequality (5.19) to hold. Then, by the comparison principle we get

$$
B_{M_{1}}\left(x, \tau+\theta_{1}\right) \leq \bar{w}(x, \tau) \quad \forall|x| \in \mathbb{R}^{N}, \tau>0 .
$$

Putting equality in the inequalities (5.22) and (5.23) we get

$$
M_{1}=c_{1} \epsilon \rho_{0}^{N}, \quad \theta_{1}=c_{2} \epsilon^{1-m} \rho_{0}^{2 s}
$$

(with $c_{1}, c_{2}$ positive constants not depending on $\varepsilon$ or $\rho_{0}$ ). We can easily see that the expressions are dimensionally correct. The constants $c_{1}$ and $c_{2}$ were defined in (5.14). In particular, $\left(M_{1}^{m-1} \theta_{1}\right)^{\beta}=c_{3} \rho_{0}$ with $c_{3}=\left(F_{1}(0) / K_{1}\right)^{-1 /(N+2 s)}$.

Since $v_{0} \leq \epsilon$ in $\mathbb{R}^{N}$, then $\bar{w}(x, \tau) \leq \epsilon$ for all $x \in \mathbb{R}^{N}, \tau>0$, and then in terms of $w(x, t)$ we obtain the following bound:

$$
0 \leq w(x, t) \leq e^{\frac{f(\delta)}{\delta} t_{0}} \epsilon \leq e^{f^{\prime}(0) t_{0}} \epsilon_{0}=\delta \quad \forall t \leq t_{0}
$$

Since $\frac{f(\delta)}{\delta} \xi \leq f(\xi)$ for $0 \leq \xi \leq \delta$, then $w$ is a subsolution of problem (1.1) in $\left[0, t_{0}\right] \times \mathbb{R}^{N}$. By the comparison principle and estimate (5.24) we obtain that at the moment $t_{0}$

$$
v\left(\cdot, t_{0}\right) \geq w\left(\cdot, t_{0}\right)=e^{\frac{f(\delta)}{\delta} t_{0}} \bar{w}\left(\cdot, \tau_{0}\right) \geq e^{\frac{f(\delta)}{\delta} t_{0}} B_{M_{1}}\left(\cdot, \tau_{0}+\theta_{1}\right) \text { in } \mathbb{R}^{N},
$$

where we use the notation $\tau_{0}=\tau\left(t_{0}\right)$ defined by $(5.17)$.

Copyright (c) by SIAM. Unauthorized reproduction of this article is prohibited. 
IIc. We will now prove that estimate (5.26) with the choices (5.25) for $M_{1}$ and $\theta_{1}$ implies the lower bound (5.11) stated in Lemma 5.3 in the case $k=1, m>1$. Indeed, we have

$$
\begin{aligned}
v\left(x, t_{0}\right) & \geq e^{\frac{f(\delta)}{\delta} t_{0}} B_{M_{1}}\left(x, \tau_{0}+\theta_{1}\right) \\
& =e^{\frac{f(\delta)}{\delta} t_{0}} M_{1}^{2 \beta s}\left(\tau_{0}+\theta_{1}\right)^{-\alpha} F_{1}\left(M_{1}^{-(m-1) \beta}\left(\tau_{0}+\theta_{1}\right)^{-\beta}|x|\right) \\
& \geq e^{\frac{f(\delta)}{\delta} t_{0}} M_{1}^{2 \beta s}\left(\tau_{0}+\theta_{1}\right)^{-\alpha} K_{2}\left(1+\left(M_{1}^{-(m-1) \beta}\left(\tau_{0}+\theta_{1}\right)^{-\beta}|x|\right)^{(N+2 s)}\right)^{-1} .
\end{aligned}
$$

Our aim now is to be able to continue this estimate until we reach a bound $v_{1}(x)$ of the form (5.10) for the same $\epsilon$ and a larger radius $\rho_{1}$. We will choose some $\rho_{1}$ and then check that the lower bound for $v\left(x, t_{0}\right)$ is larger than $\epsilon$ at $|x|=\rho_{1}$. In order to simplify the estimate of the last parenthesis in formula (5.27), we will impose the condition

$$
M_{1}^{-(m-1) \beta}\left(\tau_{0}+\theta_{1}\right)^{-\beta} \rho_{1} \geq 1
$$

which is natural since the radius $\rho_{1}$ in the iteration process will increase. Then we only need to have

$$
v\left(x, t_{0}\right) \geq\left(K_{2} / 2\right) e^{\frac{f(\delta)}{\delta} t_{0}} M_{1}^{1+2(m-1) \beta s}\left(\tau_{0}+\theta_{1}\right)^{2 \beta s} \rho_{1}^{-(N+2 s)} \geq \epsilon \quad \text { for }|x|=\rho_{1} .
$$

Notice that $M_{1}^{-(m-1) \beta}\left(\tau_{0}+\theta_{1}\right)^{-\beta} \rho_{1}=c_{3}^{-1}\left(1+\left(\tau_{0} / \theta_{1}\right)\right)^{-\beta}\left(\rho_{1} / \rho_{0}\right)$. Hence the first condition (5.28) is equivalent to

$$
\rho_{1} / \rho_{0} \geq c_{3}\left(1+\left(\tau_{0} / \theta_{1}\right)\right)^{\beta},
$$

while, taking into account that $M_{1}^{1+2(m-1) \beta s} \theta_{1}^{2 \beta s}=a_{0} / K_{1}$ and $a_{0}=\epsilon \rho_{0}^{N+2 s}$, the second, (5.29), means that

$$
\left(\rho_{1} / \rho_{0}\right)^{(N+2 s)} \leq\left(K_{2} / 2 K_{1}\right) e^{\frac{f(\delta)}{\delta} t_{0}}\left(1+\left(\tau_{0} / \theta_{1}\right)\right)^{2 \beta s} .
$$

Both conditions are compatible iff

$$
e^{\frac{f(\delta)}{\delta} t_{0}}\left(1+\left(\tau_{0} / \theta_{1}\right)\right)^{-N \beta} \geq K_{3},
$$

where $K_{3}:=2 K_{1} K_{2}^{-1} c_{3}^{N+2 s}=2 F_{1}(0) / K_{2}$. Now recall that $\theta_{1}$ depends on $\rho_{0}$ by (5.25), $\rho_{0} \geq 1$, and $\theta_{1}$ is bounded below by $\tau_{*}=\epsilon^{1-m} c_{2}$, the value for $\rho_{0}=1$. Since $m \geq 1, \epsilon<1$, then $\theta_{1} \geq \tau_{*} \geq c_{2}$. We see this condition as a way of choosing $t_{0}$. Let us find a simpler condition for $t_{0}$ such that the required inequality (5.31) holds true. To this aim, observe that $\tau_{0}=\tau\left(t_{0}\right) \leq t_{0} e^{(m-1)(f(\delta) / \delta) t_{0}}$, which can be seen by definition (5.17). Then, it will be enough for $t_{0}$ to satisfy

$$
e^{\frac{f(\delta)}{\delta} t_{0}} \geq\left(1+\frac{t_{0}}{c_{2}} e^{(m-1)(f(\delta) / \delta) t_{0}}\right)^{N \beta} K_{3} .
$$

This inequality is possible when the exponents are ordered, i.e., if $(m-1) N \beta<1$, which is true since $1-(m-1) N \beta=2 \beta$ s. In particular, we can take $t_{0}$ large enough such that

$$
e^{2 \beta s \frac{f(\delta)}{\delta} t_{0}} \geq\left(1+\frac{t_{0}}{c_{2}}\right)^{N \beta} K_{3} .
$$

Copyright $@$ by SIAM. Unauthorized reproduction of this article is prohibited. 
This last choice of $t_{0}$ is a sufficient for condition (5.31) to hold true. It is independent of $\rho_{0}$ and $\epsilon$, and this will be used below.

Once this is guaranteed, we choose the largest possible $\rho_{1}$ satisfying (5.30), which is

$$
\frac{\rho_{1}}{\rho_{0}}=\left(K_{2} / 2 K_{1}\right)^{1 /(N+2 s)} e^{\frac{f(\delta)}{(N+2 s) \delta} t_{0}}\left(1+\left(\tau_{0} / \theta_{1}\right)\right)^{2 s \beta /(N+2 s)}:=L_{0}
$$

Hence formula (5.29) takes place with equality in the second inequality.

Comments on the new radius $\boldsymbol{\rho}_{\mathbf{1}}$. Notice that $\rho_{1} \geq e^{\sigma t_{0}} \rho_{0}$ since $t_{0}$ satisfies (5.13).

IId. With this choice of $\rho_{1}$ and $t_{0}$, estimate (5.29) holds. In conclusion, we have

$$
v\left(x, t_{0}\right) \geq e^{\frac{f(\delta)}{\delta} t_{0}} B_{M_{1}}\left(x, \tau\left(t_{0}\right)+\theta_{1}\right) \geq \epsilon \quad \text { for }|x|=\rho_{1},
$$

and thus since the profile $F_{1}$ is nonincreasing we get that

$$
v\left(x, t_{0}\right) \geq \epsilon \quad \forall|x| \leq \rho_{1} .
$$

The behavior for large $|x|$ is as follows. For $|x| \geq \rho_{1}$ we have $M_{1}^{-(m-1)}\left(\tau_{0}+\theta_{1}\right)^{-\beta}|x| \geq$ 1 according to (5.29) and (5.32) we get that

$$
v\left(x, t_{0}\right) \geq \epsilon \rho_{1}^{N+2 s}|x|^{-(N+2 s)} \quad \forall|x| \geq \rho_{1} .
$$

Remark that $\rho_{0} e^{\sigma t_{0}} \leq \rho_{1}$. Finally, we define $a_{1}:=\epsilon \rho_{1}^{N+2 s}$ and thus $v\left(\cdot, t_{0}\right) \geq v_{1}(\cdot)$, where $v_{1}$ is given by the expression

$$
v_{1}(x)= \begin{cases}a_{1}|x|^{-(N+2 s)}, & |x| \geq \rho_{1} ; \\ \epsilon=a_{1} \rho_{1}^{-(N+2 s)}, & |x| \leq \rho_{1} .\end{cases}
$$

The proof is complete for $m>1$ and $k=1$. (See Figures 2 and 3 for the construction of $v_{1}$.)

III. The iteration. We are now ready to address the next delicate step. Once we have proved that $v\left(x, t_{0}\right) \geq v_{1}(x)$ for all $x \in \mathbb{R}^{N}$, where $v_{1}$ is defined above, we apply the same proof and result to obtain

$$
v\left(x, 2 t_{0}\right) \geq\left(\text { solution of KPP with initial data } v_{1}(x)\right)\left(t_{0}\right) \geq v_{2}(x),
$$

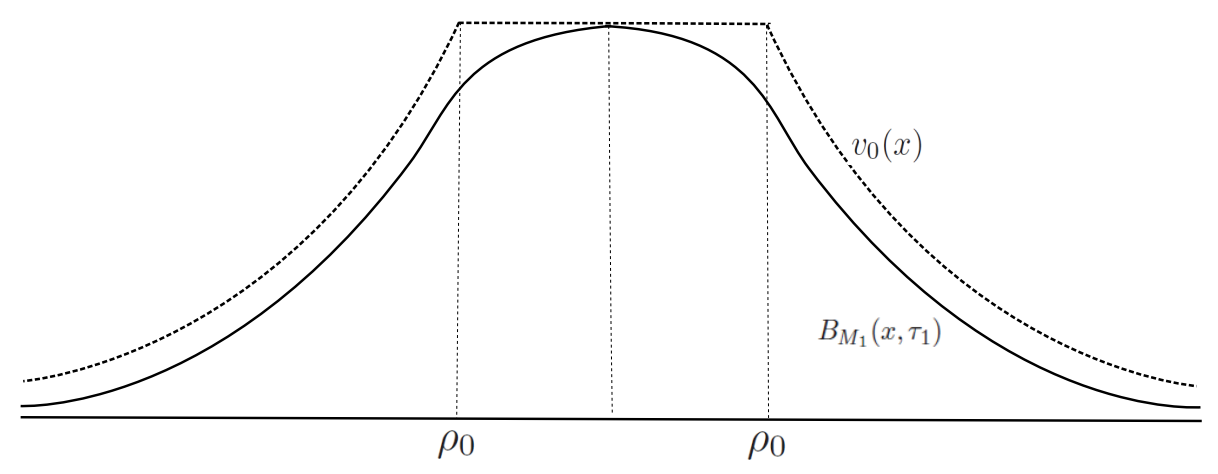

FIG. 2. Step IIb.

Copyright $@$ by SIAM. Unauthorized reproduction of this article is prohibited. 


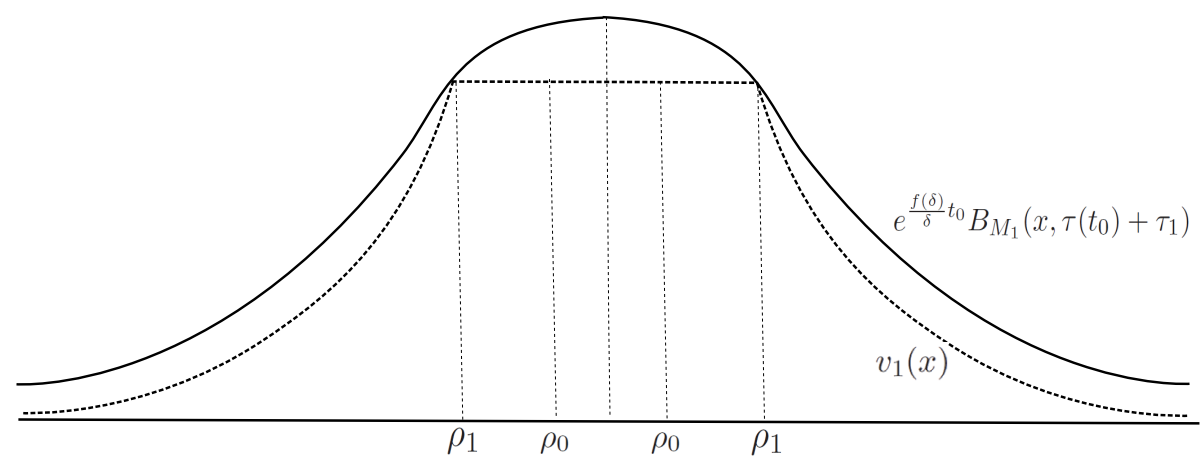

FIG. 3. Step IIc.

where $v_{2}(x)$ has the same construction as $v_{0}(x)$ and $v_{1}(x)$ but with parameters $\rho_{2}$ and $a_{2}$. Since $\rho_{1}>\rho_{0}$ the previous choice of $t_{0}$ is still valid to get to a similar conclusion. The argument continues for all $k=3,4, \ldots$.

Let us check more closely the quantitative part of the iteration in order to get an improvement. In the process we keep $\epsilon$ fixed but we replace $\rho_{0}$ by $\rho_{k}, k \geq 1$, so that the formula (5.32) becomes

$$
\frac{\rho_{k+1}}{\rho_{k}}=L_{k}:=\left(K_{2} / 2 K_{1}\right)^{1 /(N+2 s)} e^{\frac{f(\delta)}{(N+2 s) \delta} t_{0}}\left(1+\left(\tau_{0} / \theta_{1}\left(\rho_{k}\right)\right)\right)^{2 s \beta /(N+2 s)} .
$$

Now, if we are given some $\sigma<\sigma_{2}=f^{\prime}(0) /(N+2 s)$, we define

$$
L_{\infty}=\left(K_{2} / 2 K_{1}\right)^{1 /(N+2 s)} e^{\frac{f(\delta)}{(N+2 s) \delta} t_{0}}
$$

and impose that $L_{\infty} \geq e^{\sigma t_{0}}$ by changing the definition of $t_{0}$. (Note that this is compatible.) Then we have $L_{k} \geq L_{\infty} \geq e^{\sigma t_{0}}$ for every $k$, so that as $k \rightarrow \infty$ we have $\rho_{k} \rightarrow \infty$ in an exponential way. The conditions we put on $\delta$ and $t_{0}$ can be summarized in (5.12) and (5.13), and they are independent on the parameters $\theta_{k}, \rho_{k}$ of the iteration. This ends the proof for $m>1$.

Proof of Lemma 5.3 in the case $m<1$. The outline of the proof is similar to that for case $m>1$. We explain the differences that appear and that are not technically trivial.

I. Preliminary choices. From the beginning we fix $\sigma \in\left(0, \sigma_{2}\right)$ and $\rho_{0} \gg 1$. We take $\delta \in(0,1)$ small enough such that

$$
\frac{f(\delta)}{(N+2 s) \delta}>\sigma
$$

We take $t_{0}$ large enough such that

$$
\frac{K_{2}}{2} e^{\frac{f(\delta)}{\delta} t_{0}} \geq F_{1}(0) 2^{\beta N}, \quad\left(K_{2} / 2 K_{1}\right)^{1 /(N+2 s)} e^{\frac{f(\delta)}{(N+2 s) \delta} t_{0}} \geq e^{\sigma t_{0}} .
$$

Notice that (i) $\delta$ depends only on $\sigma$; (ii) $t_{0}$ depends only on $\sigma, \delta$ and some constants appearing in the characterization of the Barenblatt function.

In this case we introduce the new time $\tau$ via

$$
\tau=\frac{1}{(1-m) f(\delta) / \delta}\left[1-e^{-(1-m) \frac{f(\delta)}{\delta} t}\right] \text { if } m<1
$$

Copyright $@$ by SIAM. Unauthorized reproduction of this article is prohibited. 
Therefore, for each $t$ we have a new bounded time $\tau(t) \leq \tau_{\infty}=1 /((1-m) f(\delta) / \delta)$. For $t=t_{0}$ we denote the corresponding $\tau\left(t_{0}\right)=: \tau_{0}$.

Next, we define $\epsilon_{0}$ by $e^{(f(\delta) / \delta) t_{0}} \epsilon_{0}=\delta$. Fix $0<\epsilon<\epsilon_{0}$. At this moment, we ensure that the first radius $\rho_{0}$ appearing in the proof is large enough; more exactly, we ask $\rho_{0}$ to satisfy

$$
\frac{1}{(1-m) f(\delta) / \delta} \epsilon^{-(1-m)} \leq c_{2} \rho_{0}^{2 s}
$$

This condition says that $\rho_{0}=\rho_{0}(\epsilon)$ is sufficiently large depending on $\epsilon$.

These values are set before starting the proof of the first step $k=1$. Hence, these values will be the same during the iteration process.

II. First step $k=1$. We consider the initial data $v_{0}$ defined by (5.10). We take $M_{1}$ and $\theta_{1}$ satisfying conditions (5.22) and (5.23):

$$
M_{1}^{2 \beta s} \theta_{1}^{-\alpha} F_{1}(0)=\epsilon, \quad K_{1} M_{1}^{1+2 \beta s(m-1)} \theta_{1}^{2 \beta s}=\epsilon \rho_{0}^{N+2 s} .
$$

Therefore $M_{1}=c_{1} \epsilon \rho_{0}^{N}$ and $\theta_{1}=c_{2} \epsilon^{1-m} \rho_{0}^{2 s}$. In what follows, we will need $\rho_{0}$ large enough such that

$$
\tau_{0} \leq \theta_{1}
$$

Hence, it is sufficient to have $\tau_{\infty} \leq \theta_{1}$ which is satisfied for $\rho_{0}=\rho_{0}(\epsilon)$ large enough according to the previous choice (5.34).

Then, at point IIc of the previous proof we have

$$
\begin{aligned}
v\left(x, t_{0}\right) & \geq e^{\frac{f(\delta)}{\delta} t_{0}} M_{1}^{2 \beta s}\left(\tau_{0}+\theta_{1}\right)^{-\alpha} F_{1}\left(M_{1}^{-(m-1) \beta}\left(\tau_{0}+\theta_{1}\right)^{-\beta}|x|\right) \\
& \geq e^{\frac{f(\delta)}{\delta} t_{0}} M_{1}^{2 \beta s}\left(\tau_{0}+\theta_{1}\right)^{-\alpha} K_{2}\left(1+\left(M_{1}^{-(m-1) \beta}\left(\tau_{0}+\theta_{1}\right)^{-\beta}|x|\right)^{(N+2 s)}\right)^{-1} .
\end{aligned}
$$

Our purpose is to find suitable $\rho_{1}$ such that $v\left(x, t_{0}\right) \geq v_{1}(x)$ for all $x \in \mathbb{R}^{N}$, where $v_{1}(x)$ is defined as

$$
v_{1}(x)= \begin{cases}a_{1}|x|^{-(N+2 s)}, & |x| \geq \rho_{1}, \\ \epsilon=a_{1} \rho_{1}^{-(N+2 s)}, & |x| \leq \rho_{1},\end{cases}
$$

Since the profile $F_{1}$ is nonincreasing, the idea is to find $\rho_{1}$ such that when $|x|=\rho_{1}$

$$
\begin{aligned}
v\left(x, t_{0}\right) & \geq e^{\frac{f(\delta)}{\delta} t_{0}} M_{1}^{2 \beta s}\left(\tau_{0}+\theta_{1}\right)^{-\alpha} K_{2}\left(1+\left(M_{1}^{-(m-1) \beta}\left(\tau_{0}+\theta_{1}\right)^{-\beta} \rho_{1}\right)^{(N+2 s)}\right)^{-1} \\
& \geq a_{1} \rho_{1}^{-(N+2 s)}=\epsilon .
\end{aligned}
$$

In particular if we take

$$
M_{1}^{-(m-1) \beta}\left(\tau_{0}+\theta_{1}\right)^{-\beta} \rho_{1} \geq 1,
$$

then for $|x|=\rho_{1}$

$$
\begin{aligned}
v\left(x, t_{0}\right) & \geq e^{\frac{f(\delta)}{\delta} t_{0}} M_{1}^{2 \beta s}\left(\tau_{0}+\theta_{1}\right)^{-\alpha} K_{2}\left(2\left(M_{1}^{-(m-1) \beta}\left(\tau_{0}+\theta_{1}\right)^{-\beta} \rho_{1}\right)^{(N+2 s)}\right)^{-1} \\
& =\frac{K_{2}}{2} e^{\frac{f(\delta)}{\delta} t_{0}} M_{1}^{2 \beta s+(m-1) \beta(N+2 s)}\left(\tau_{0}+\theta_{1}\right)^{-\alpha+\beta(N+2 s)} \rho_{1}^{-(N+2 s)} \\
& =\frac{K_{2}}{2} e^{\frac{f(\delta)}{\delta} t_{0}} M_{1}^{1+2 \beta s(m-1)}\left(\tau_{0}+\theta_{1}\right)^{2 \beta s} \rho_{1}^{-(N+2 s)} .
\end{aligned}
$$

Copyright $@$ by SIAM. Unauthorized reproduction of this article is prohibited. 
We take $\rho_{1}$ the largest value satisfying

$$
\left(K_{2} / 2\right) e^{\frac{f(\delta)}{\delta} t_{0}} M_{1}^{1+2 \beta s(m-1)}\left(\tau_{0}+\theta_{1}\right)^{2 \beta s} \rho_{1}^{-(N+2 s)} \geq \epsilon,
$$

that is, we take $\rho_{1}$ such that

$$
\frac{K_{2}}{2} e^{\frac{f(\delta)}{\delta} t_{0}} M_{1}^{1+2 \beta s(m-1)}\left(\tau_{0}+\theta_{1}\right)^{2 \beta s} \rho_{1}^{-(N+2 s)}=\epsilon .
$$

Then for $|x|=\rho_{1}$ we have

$$
v\left(x, t_{0}\right) \geq \epsilon=a_{1} \rho_{1}^{-(N+2 s)}, \quad a_{1}:=\frac{K_{2}}{2} e^{\frac{f(\delta)}{\delta} t_{0}} M_{1}^{1+2 \beta s(m-1)}\left(\tau_{0}+\theta_{1}\right)^{2 \beta s} .
$$

For $|x| \leq \rho_{1}$ we have $v\left(x, t_{0}\right) \geq \epsilon$ and for $|x| \geq \rho_{1}$ we have $v\left(x, t_{0}\right) \geq a_{1}|x|^{-(N+2 s)}$ justified as in the previous case $m>1$.

It remains to check that conditions (5.37) and (5.38) are compatible, that is, we need $t_{0}$ such that

$$
\frac{K_{2}}{2} e^{\frac{f(\delta)}{\delta} t_{0}} M_{1}^{1+2 \beta s(m-1)}\left(\tau_{0}+\theta_{1}\right)^{2 \beta s} \epsilon^{-1}=\rho_{1}^{N+2 s}>\left(\left(M_{1}^{(m-1) \beta}\left(\tau_{0}+\theta_{1}\right)^{\beta}\right)^{N+2 s} .\right.
$$

This is equivalent to

$$
\frac{K_{2}}{2} e^{\frac{f(\delta)}{\delta} t_{0}} M_{1}^{2 \beta s} \theta_{1}^{-\beta N} \epsilon^{-1} \geq\left(1+\frac{\tau_{0}}{\theta_{1}}\right)^{\beta N}
$$

According to the definition of $M_{1}$ and $\theta_{1}$ this is rewritten as

$$
\frac{K_{2}}{2} e^{\frac{f(\delta)}{\delta} t_{0}} \geq F_{1}(0)\left(1+\frac{\tau_{0}}{\theta_{1}}\right)^{\beta N}
$$

Since $\tau_{0} / \theta_{1} \leq 1$, then a sufficient condition for $t_{0}$ would be

$$
\frac{K_{2}}{2} e^{\frac{f(\delta)}{\delta} t_{0}} \geq F_{1}(0) 2^{\beta N} .
$$

Notice that this condition on $t_{0}$ is independent on $\epsilon$ and $\rho_{0}$. Hence $t_{0}$ is the same in the iteration, that is, done as before.

Comments on the new radius $\boldsymbol{\rho}_{\mathbf{1}}$. By the definition formula (5.38) we have

$$
\left(\frac{\rho_{1}}{\rho_{0}}\right)^{N+2 s}=\frac{K_{2}}{2 K_{1}} e^{\frac{f(\delta)}{\delta} t_{0}}\left(1+\frac{\tau_{0}}{\theta_{1}}\right)^{2 \beta s} .
$$

Now, if $t_{0}$ is such that $\left(K_{2} / 2 K_{1}\right) e^{\frac{f(\delta)}{\delta} t_{0}} \geq e^{(N+2 s) \sigma t_{0}}$, then we get that

$$
\rho_{1} / \rho_{0} \geq e^{\sigma t_{0}} .
$$

III. The iteration. We point out that for the next step $k=2$, the first radius is $\rho_{1}$. The value of $\rho_{1}$ was defined in (5.38) and satisfies $\rho_{1}>\rho_{0}$. Hence, $\rho_{1}$ satisfies the preliminary condition (5.34) and is a good candidate for the initial radius.

The rest of the proof follows as in the case $m>1$.

Remark. We summarize the results proved so far as follows: for small $\epsilon$ fixed, we found a $\rho_{0}$ sufficiently large (depending on $\epsilon$ ) such that the line $v(x, t)=\epsilon$ propagates 
with exponential speed $\sigma$. When taking a smaller $\epsilon$ and a larger value of $\rho_{0}$, the proof also works. Hence, the result of exponential propagation is true for all small $\epsilon<\epsilon_{0}$.

Proof of Lemma 5.3 in the case $m=1$. No change of the time variable is needed in this case, that is, $\tau=t$. The proof follows similarly to the case $m>1$. We do not give more details here, since the result for $m=1$ has been proved in [12].

Lemma 5.4 (expansion of uniform positivity for all times). Let $N \geq 1, s \in(0,1)$, $m_{1}<m, f$ satisfying (1.2) and $\sigma \in\left(0, \sigma_{2}\right)$. Let $t_{0}>0$ from Lemma 5.3. Then for every measurable initial datum $u_{0}$ with $0 \leq u_{0} \leq 1, u_{0} \neq 0$, there exist $\epsilon \in(0,1)$ and $b>0$ (both depending on $u_{0}$ ) such that the solution $u$ of problem (1.1) with initial datum $u(0, \cdot)=u_{0}$ satisfies

$$
u(x, t) \geq \epsilon \forall t \geq t_{0} \text { and }|x| \leq b e^{\sigma t} .
$$

Proof. Let $t_{0}$ defined in Lemma 5.3. Then by Lemma 5.2 there exist $\epsilon>0, a_{0}>0$, $\rho_{0}>1$ such that $u(x, t)$ is bounded from below by a function $v_{0}$ with the long tail behavior at infinity

$$
u(x, t) \geq v_{0}(x):= \begin{cases}a_{0}|x|^{-(N+2 s)}, & |x| \geq \rho_{0}, \\ \epsilon=a_{0} \rho_{0}^{-(N+2 s)}, & |x| \leq \rho_{0},\end{cases}
$$

for all $x \in \mathbb{R}^{N}, t \in\left[t_{0}, 2 t_{0}\right]$. In this way $v_{0}$ can be taken as the initial datum (5.10) in Lemma 5.3. If necessary, we make $a_{0}$ smaller and $\rho_{0}$ larger to fit in the context of Lemma 5.3. We recall the necessary conditions: $\epsilon \leq \epsilon_{0}$ in (5.15) and $\rho_{0} \geq \rho(\epsilon)$ in (5.34).

Therefore, by applying Lemma 5.3, the solution $u$ will be raised an $\epsilon$ at a large time $\tau_{0}+t_{0}$ and this holds true for all $\tau_{0} \in\left[t_{0}, 2 t_{0}\right]$. More exactly, by (5.11), for every $k=0,1,2, \ldots$ one has

$$
u\left(x, \tau_{0}+k t_{0}\right) \geq \epsilon \forall|x| \leq e^{\sigma k t_{0}} \rho_{0}, \tau_{0} \in\left[t_{0}, 2 t_{0}\right],
$$

which is rewritten as

$$
u(x, t) \geq \epsilon \forall|x| \leq e^{\sigma k t_{0}} \rho_{0}, \quad t \in\left[(k+1) t_{0},(k+2) t_{0}\right] .
$$

But for $t \in\left[(k+1) t_{0},(k+2) t_{0}\right]$ we get $e^{\sigma k t_{0}}=e^{\sigma k t_{0}-\sigma t} e^{\sigma t} \geq e^{-2 \sigma t_{0}} e^{\sigma t}$ and then (5.39) implies, in particular, that

$$
u(x, t) \geq \epsilon \forall|x| \leq e^{-2 \sigma t_{0}} e^{\sigma t} \rho_{0}, \quad t \in\left[(k+1) t_{0},(k+2) t_{0}\right] .
$$

Since the union the intervals $\left[(k+1) t_{0},(k+2) t_{0}\right]$ with $k=0,1,2, \ldots$ cover all $\left[t_{0}, \infty\right)$, we deduce that

$$
u(x, t) \geq \epsilon \quad \text { if } \quad t \geq t_{0} \text { and }|x| \leq \rho_{0} e^{-\sigma 2 t_{0}} e^{\sigma t} .
$$

The proof of the lemma follows by denoting $b=\rho_{0} e^{-\sigma 2 t_{0}}$.

Lemma 5.5. Let $N \geq 1, s \in(0,1), m>m_{1}$, and $f$ satisfying (1.2). Let $\sigma_{2}=\frac{f^{\prime}(0)}{N+2 s}$. Let $u$ be a solution of problem $(1.1)$ with initial datum $0 \leq u_{0}(\cdot) \leq 1$, $u_{0} \neq 0$. Then for every $\sigma<\sigma_{2}$ there exist $\epsilon \in(0,1)$ and $\underline{t}>0$ such that

$$
u(x, t) \geq \epsilon \quad \forall t \geq \underline{t} \text { and }|x| \leq e^{\sigma t} .
$$

Proof. We apply Lemma 5.4 with $\sigma$ replaced by $\sigma^{\prime} \in\left(\sigma, \sigma_{2}\right)$. Since $e^{\sigma t} \leq b e^{\sigma^{\prime} t}$ for $t$ large, where $b$ is the constant in the statement of Lemma 5.4, we deduce that

$$
u(x, t) \geq \epsilon \text { for } t \geq \underline{t} \text { and }|x| \leq e^{\sigma t} .
$$

Copyright (c) by SIAM. Unauthorized reproduction of this article is prohibited. 
5.2. Case $\boldsymbol{m}_{\boldsymbol{c}}<\boldsymbol{m}<\boldsymbol{m}_{\mathbf{1}}$. In a similar way, we can prove the convergence to 1 on the inner sets also in the range of parameters $m_{c}<m<m_{1}$.

Proposition 5.6. Let $N \geq 1, s \in(0,1), m_{c}<m<m_{1}$, $f$ satisfying (1.2). Let $\sigma_{1}=\frac{1-m}{2 s} f^{\prime}(0)$. Let $u$ be a solution of problem (1.1) with initial datum $0 \leq u_{0}(\cdot) \leq 1$, $u_{0} \neq 0$. Then for every $\sigma \in\left(0, \sigma_{1}\right), u(x, t) \rightarrow 1$ uniformly on $\left\{|x| \leq e^{\sigma t}\right\}$ as $t \rightarrow \infty$.

Proof. We argue in a similar way as in the case $m>m_{1}$ proved in Proposition 5.1. The difference appears when obtaining the positivity on inner sets. To this aim, we start with nontrivial initial data $0 \leq u_{0} \leq 1$ and we prove the analogue of Lemma 5.4. The key ingredient is to use the quantitative lower estimates for the solution $\underline{u}(x, t)$ of the fractional fast diffusion equation stated in Theorem 2.6 to obtain an estimate of the form

$$
\underline{u}(x, t) \geq v_{0}(x) \quad \forall t \in\left[t_{0}, 2 t_{0}\right], x \in \mathbb{R}^{N},
$$

where $v_{0}(x)$ is defined as

$$
v_{0}(x)= \begin{cases}a_{0}|x|^{-2 s /(1-m)}, & |x| \geq \rho_{0}, \\ \epsilon=a_{0} \rho_{0}^{-2 s /(1-m)}, & |x| \leq \rho_{0} .\end{cases}
$$

Afterward, we can prove an analogue result to Lemma 4.1 starting with initial data of the form (5.40). Since the Barenblatt solution has a long tail decay of the form $|x|^{-2 s /(1-m)}$, then we find $M_{1}>0$ and $\theta_{1}>0$ such that

$$
v_{0}(x) \geq B_{M_{1}}\left(x, \theta_{1}\right) \quad \forall x \in \mathbb{R}^{N} .
$$

6. The linear diffusion problem. We will need a number of facts about the linear diffusion equation for $0<s<1$,

$$
U_{t}+(-\Delta)^{s} U=0 \quad \text { for } x \in \mathbb{R}^{N} \text { and } t>0
$$

This problem has been studied, mainly by probabilists [2, 7], (see also [25]), and many results are known. When considering initial data $U_{0} \in L^{1}\left(\mathbb{R}^{N}\right)$, or more generally,

$$
U(0, x)=U_{0}(x) \quad \text { for } x \in \mathbb{R}^{N},
$$

the solution of problem (6.1)-(6.2) has the integral representation

$$
U(x, t)=\int_{\mathbb{R}^{N}} K_{s}(x-z, t) U_{0}(z) d z,
$$

where the kernel $K_{s}$ has Fourier transform $\widehat{K}_{s}(\xi, t)=e^{-|\xi|^{2 s} t}$. If $s=1$, the function $K_{1}(x, t)$ is the Gaussian heat kernel.

6.1. The fundamental solution. Further results on the asymptotics for large $|\boldsymbol{x}|$. We need some detailed information on the behavior of the kernel $K_{s}(x, t)$ for $0<s<1$. In the particular case $s=1 / 2$, the kernel is explicit, given by the formula

$$
K_{1 / 2}(x, t)=C_{N} t\left(|x|^{2}+t^{2}\right)^{-(N+1) / 2} .
$$

In general, we know that the kernel $K_{s}(x, t)$ is the fundamental solution of problem (6.1), that is, $K_{s}(x, t)$ solves the problem with initial data the Delta function

$$
\lim _{t \rightarrow 0} K_{s}(x, t)=\delta(x) .
$$

Copyright (C) by SIAM. Unauthorized reproduction of this article is prohibited. 
It is known that the kernel $K_{s}$ has the form

$$
K_{s}(x, t)=t^{-N / 2 s} f\left(|x| t^{-1 / 2 s}\right)
$$

for some profile function, $f(r)$, that is positive and decreasing, and behaves at infinity like $f(r) \sim r^{-(N+2 s)}$; cf. [8].

We perform now a further analysis of the properties of the fundamental solution. Our aim is to prove the following result.

Proposition 6.1. For every $s \in(0,1)$, the fundamental solution $K_{s}(x, t)$ of problem (6.1) is a increasing function in time

$$
\frac{d}{d t} K_{s}(x, t) \geq 0 \quad \text { for all large values of }|x| / t^{1 / 2 s} .
$$

This property is known to be satisfied for the fundamental solution of various types of diffusion equations of evolution type: the Gaussian profile for the heat equation, the Barenblatt solution for the fast diffusion equation.

The analysis of the derivative $\frac{d}{d t} K_{s}(x, t)$ involves not only the characterization of the profile $f$ for large $r$, but also a similar property for the derivative $f^{\prime}$. In fact, we will prove that $f(r)$ and $r f^{\prime}(r)$ have the same behavior for large arguments. This is due to the power decay property of the profile $f$.

We recall that this property is clearly true in the explicit case $s=1 / 2$ where $f(s)=\left(1+s^{2}\right)^{-(N+1) / 2}$. But it is not true in the limit $s \rightarrow 1$, i.e., in the case of the Gaussian profile of the heat equation $G(s)=e^{-s^{2} / 4}$. Indeed, we cannot obtain the same behavior for $G(s)$ and $s G^{\prime}(s)$ since in this case the profile has an exponential expression.

Proof of the proposition. We recall that $[8]$

$$
K_{s}(x, t)=t^{-\frac{N}{2 s}} f_{2 s}\left(1, t^{-\frac{1}{2 s}}|x|\right),
$$

where $f_{2 s}(1, x)$ is a continuous strictly positive function on $\mathbb{R}^{N}$ of radial type, which is explicitly given by the expression

$$
\begin{aligned}
f_{2 s}(1, x) & =\left[(2 \pi)^{N / 2}|x|^{\frac{N}{2}-1}\right]^{-1} \int_{0}^{\infty} e^{-\omega^{2 s}} \omega^{\frac{N}{2}} J_{\nu}(|x| \omega) d \omega \\
& =\frac{1}{(2 \pi)^{N / 2}|x|^{N}} \int_{0}^{\infty} e^{-\left(\frac{\omega}{|x|}\right)^{2 s}} \omega^{\frac{N}{2}} J_{\nu}(\omega) d \omega, \quad \nu=(N-2) / 2,
\end{aligned}
$$

where $J_{\mu}$ denotes the Bessel function of first kind of order $\mu$. For simplicity, we denote $f(r)=f_{2 s}(1, x), r=|x|$ since $f_{2 s}(1, \cdot)$ is a radial function:

$$
f(r)=\frac{1}{(2 \pi)^{N / 2}} r^{-N} \int_{0}^{\infty} e^{-\left(\frac{\omega}{r}\right)^{2 s}} \omega^{\frac{N}{2}} J_{\nu}(\omega) d \omega, \quad \nu=(N-2) / 2 .
$$

Next, we prove an intermediate result, concerning the behavior of the derivative $f^{\prime}$.

Lemma 6.2. Let $s \in(0,1)$ and let $f(r)=f_{2 s}(1, x)$ be defined by $(6.5)$. Then

$$
\lim _{r \rightarrow \infty} r^{N+2 s}\left(N f(r)+r f^{\prime}(r)\right)=-s^{2} 2^{2 s+1} \frac{1}{\pi^{1+N / 2}}(\sin \pi s) \Gamma(s) \Gamma\left(s+\frac{N}{2}\right) .
$$

In particular, we prove that $r f^{\prime}(r) \sim-r^{-(N+2 s)}$ for large $r$. 
Proof. We compute the derivative with respect to $r$

$$
f^{\prime}(r)=\frac{1}{(2 \pi)^{N / 2}} r^{-N-1} \int_{0}^{\infty}\left(-N+2 s\left(\frac{\omega}{r}\right)^{2 s}\right) e^{-\left(\frac{\omega}{r}\right)^{2 s}} \omega^{\frac{N}{2}} J_{\nu}(\omega) d \omega .
$$

Therefore

$$
r f^{\prime}(r)=-N f(r)+\frac{1}{(2 \pi)^{N / 2}} r^{-N} \int_{0}^{\infty} 2 s\left(\frac{\omega}{r}\right)^{2 s} e^{-\left(\frac{\omega}{r}\right)^{2 s}} \omega^{\frac{N}{2}} J_{\nu}(\omega) d \omega=(\mathrm{I})+(\mathrm{II}),
$$

where (I) $=-N f(r)$, and (II) is given by

$$
(\mathrm{II})=2 s \frac{1}{(2 \pi)^{N / 2}} r^{-(N+2 s)} \int_{0}^{\infty} e^{-\left(\frac{\omega}{r}\right)^{2 s}} \omega^{2 s+\frac{N}{2}} J_{\nu}(\omega) d \omega .
$$

According to formula (7.4), we can write

$$
\omega J_{\frac{N}{2}-1}(\omega)=N J_{\frac{N}{2}}(\omega)-\omega J_{\frac{N}{2}+1}(\omega),
$$

and therefore

$$
\begin{aligned}
(\mathrm{II})= & 2 N s \frac{1}{(2 \pi)^{N / 2}} r^{-(N+2 s)} \int_{0}^{\infty} e^{-\left(\frac{\omega}{r}\right)^{2 s}} \omega^{2 s+\frac{N}{2}-1} J_{\frac{N}{2}}(\omega) d \omega \\
& -2 s \frac{1}{(2 \pi)^{N / 2}} r^{-(N+2 s)} \int_{0}^{\infty} e^{-\left(\frac{\omega}{r}\right)^{2 s}} \omega^{2 s+\frac{N}{2}} J_{\frac{N}{2}+1}(\omega) d \omega .
\end{aligned}
$$

Then, according to Pólya (see Blumenthal and Geetor [8])

$$
\lim _{r \rightarrow \infty} \int_{0}^{\infty} e^{-\left(\frac{\omega}{r}\right)^{2 s}} \omega^{2 s+\frac{N}{2}-1} J_{\frac{N}{2}}(\omega) d \omega=\frac{2}{\pi} \sin \pi s \int_{0}^{\infty} \omega^{2 s+\frac{N}{2}-1} K_{\frac{N}{2}}(\omega) d \omega
$$

and

$$
\lim _{r \rightarrow \infty} \int_{0}^{\infty} e^{-\left(\frac{\omega}{r}\right)^{2 s}} \omega^{2 s+\frac{N}{2}} J_{\frac{N}{2}+1}(\omega) d \omega=\frac{2}{\pi} \sin \pi s \int_{0}^{\infty} \omega^{2 s+\frac{N}{2}} K_{\frac{N}{2}+1}(\omega) d \omega .
$$

Here the functions $K_{\mu}$ are described in the paper of Erdélyi et al. [18] (not to be confused with $\left.K_{s}(x, t)\right)$. Moreover [18, p. 51], we have

$$
\begin{aligned}
& L_{1}=\int_{0}^{\infty} \omega^{2 s+\frac{N}{2}-1} K_{\frac{N}{2}}(\omega) d \omega=2^{2 s+\frac{N}{2}-2} \Gamma\left(s+\frac{N}{2}\right) \Gamma(s) . \\
& L_{2}=\int_{0}^{\infty} \omega^{2 s+\frac{N}{2}} K_{\frac{N}{2}+1}(\omega) d \omega=2^{2 s+\frac{N}{2}-1} \Gamma\left(s+\frac{N}{2}+1\right) \Gamma(s) .
\end{aligned}
$$

Therefore,

$$
\lim _{r \rightarrow \infty} r^{N+2 s}\left(r f^{\prime}(r)+N f(r)\right)=-2 s C_{1}(N, s)
$$

where

$$
C_{1}(N, s):=s 2^{2 s} \frac{1}{\pi^{1+N / 2}}(\sin \pi s) \Gamma(s) \Gamma\left(s+\frac{N}{2}\right) .
$$

If we write this result as

$$
r^{N-1}\left(r f^{\prime}(r)+N f(r)\right) \sim-2 s C_{1}(N, s) r^{-2 s-1}
$$

Copyright (c) by SIAM. Unauthorized reproduction of this article is prohibited. 
by integrating we obtain $r^{N} f(r) \sim C_{1}(N, s) r^{-2 s}$, that is,

$$
f(r) \sim C_{1}(N, s) r^{-(N+2 s)},
$$

which is exactly the result proved in [8]. Moreover, we obtain that

$$
\lim _{r \rightarrow \infty} r^{N+2 s} r f^{\prime}(r)=-(N+2 s) C_{1}(N, s),
$$

that is,

$$
r f^{\prime}(r) \sim-r^{-(N+2 s)} \quad \text { for large } r .
$$

We complete the proof of Proposition 6.1 on the behavior of the fundamental solution for large values of $\eta=|x| t^{-1 / 2 s}$.

Proof. The fundamental solution is given by

$$
K_{s}(x, t)=t^{-\frac{N}{2 s}} f\left(t^{-\frac{1}{2 s}}|x|\right) .
$$

We compute the derivative in the $t$ variable. According to the scaling formula (6.4) we obtain

$$
\begin{aligned}
\frac{d}{d t} K_{s}(x, t) & =-\frac{N}{2 s} t^{-\frac{N}{2 s}-1} f\left(t^{-\frac{1}{2 s}}|x|\right)-\frac{1}{2 s} t^{-\frac{N}{2 s}-\frac{1}{2 s}-1}|x| f^{\prime}\left(t^{-\frac{1}{2 s}}|x|\right) \\
& =-\frac{1}{2 s} t^{-\frac{N}{2 s}-1}\left[N f(\eta)+\eta f^{\prime}(\eta)\right], \quad \eta=t^{-\frac{1}{2 s}}|x|
\end{aligned}
$$

By Lemma 6.2 we know that

$$
N f(\eta)+\eta f^{\prime}(\eta) \sim-2 s C_{1}(N, s) \eta^{-(N+2 s)} \quad \text { for large } \eta,
$$

where $C_{1}(N, s)$ is a positive constant given by formula (6.6). Therefore,

$$
\frac{d}{d t} K_{s}(x, t) \sim t^{-\frac{N}{2 s}-1} C_{1}(N, s) \eta^{-(N+2 s)}=C_{1}(N, s)|x|^{-(N+2 s)} \quad \text { for large } \eta .
$$

6.2. Self-similar solutions of the linear diffusion problem. We study the existence, uniqueness, and properties of self-similar solutions of the form

$$
U(x, t)=t^{\alpha_{1}} F\left(t^{\beta_{1}}|x|\right)
$$

of the linear problem

$$
\begin{cases}U_{t}+(-\Delta)^{s} U=0 & \text { for } x \in \mathbb{R}^{N} \text { and } t>0, \\ U(0, x)=U_{0}(x)=C|x|^{\gamma} & \text { for } x \in \mathbb{R}^{N},\end{cases}
$$

where $C>0$, and $0<\gamma<2 s$ is given. The constants $\alpha_{1}, \beta_{1} \in \mathbb{R}$ will be determined such that $U(x, t)$ is a self-similar solution of problem (6.8).

Existence of a solution $U$ to Problem (6.8) follows from the representation formula (6.3) since $K_{s}(x-z, t) u_{0}(z) \sim|z|^{-(N+2 s-\gamma)}$ for large $|z|$, where $\gamma<2 s$, and then $K_{s}(x-z, t) u_{0}(z)$ is integrable away from the origin.

Let $\eta=t^{\beta_{1}}|x|$. Then,

$$
\begin{aligned}
U_{t}(x, t) & =\alpha_{1} t^{\alpha_{1}-1} F(\eta)+\beta_{1} t^{\alpha_{1}-1} \eta F^{\prime}(\eta), \\
(-\Delta)^{s} U(x, t) & =t^{\alpha_{1}}(-\Delta)^{s}\left(F\left(t^{\beta_{1}}|x|\right)\right)=t^{\alpha_{1}} t^{2 \beta_{1} s}(-\Delta)^{s} F(\eta) .
\end{aligned}
$$

We obtain a first relation on the parameters: $\alpha_{1}-1=\alpha_{1}+2 \beta_{1} s$, and then $\beta_{1}=-\frac{1}{2 s}$.

Copyright $@$ by SIAM. Unauthorized reproduction of this article is prohibited. 
Equation. The profile $F$ satisfies the equation

$$
\alpha_{1} F(\eta)+\beta_{1} \eta F^{\prime}(\eta)+(-\Delta)^{s} F(\eta)=0 .
$$

Self-similarity condition. The equation is invariant under transformations of the form

$$
T_{\lambda} U(x, t)=\lambda^{-\alpha_{1}} U\left(\lambda^{-\beta_{1}} x, \lambda t\right) .
$$

Therefore we impose $U=T_{\lambda} U$. We apply this to the initial data

$$
T_{\lambda} U(x, 0)=\lambda^{-\alpha_{1}} U\left(\lambda^{-\beta_{1}} x, 0\right)=\lambda^{-\alpha_{1}-\beta_{1} \gamma}|x|^{\gamma}
$$

and then $\alpha_{1}=-\gamma \beta_{1}$. We obtain the exact value of the similarity exponents

$$
\alpha_{1}=\frac{\gamma}{2 s}, \quad \beta_{1}=-\frac{1}{2 s} .
$$

Notice that $\alpha_{1}>0$ and $\beta_{1}<0$. As a solution of the linear problem (6.8), $U(x, t)$ can be computed as a convolution with the kernel $K_{s}(\cdot, t)$,

$$
U(x, t)=\left(K_{s}(\cdot, t) \star U_{0}\right)(x)=\int_{\mathbb{R}^{N}} K_{s}(y, t) U_{0}(x-y) d y .
$$

Since the initial data is a radial function $U_{0}(x)=|x|^{\gamma}$, then by the properties of the kernel $K_{s}, U$ will also be a radial function, and therefore the profile $F$ is radial.

Lemma 6.3 (properties of the profile). The profile $F$ is monotone nondecreasing and it satisfies $\eta F^{\prime} \leq c_{2} F$ for all $\eta \geq 0$.

Proof. I. Monotonicity property. In order to prove the positivity of $F$ we will make use of the Alexandrov symmetry principle and we prove that $U(x, t)$ is radially nondecreasing in the space variable $x \in \mathbb{R}^{N}$.

We start with nondecreasing radial initial data $U_{0}(x)=|x|^{\gamma}$. We approximate $U_{0}$ with a sequence of radially symmetric and bounded functions $U_{0 n} \in L^{\infty}\left(\mathbb{R}^{N}\right)$ such that $U_{0 n}(r) \rightarrow C n^{\gamma}$ as $r \rightarrow \infty$ and $v_{0 n}(r)=C n^{\gamma}-U_{0 n}(r) \in L^{1}\left(\mathbb{R}^{N}\right)$. Let $v_{n}$ the solution of problem (6.8) with initial datum $v_{0 n}$. We may apply the Alexandrov symmetry principle (which we explain in detail below) to $v_{n}$ to conclude that it is radially symmetric and nonincreasing w.r.t. the space variable. We then put $U_{n}(x, t)=C n^{\gamma}-v_{n}(x, t)$, which is radially symmetric and increasing and solves (6.8) with initial datum $U_{0 n}$. We pass now to the limit $n \rightarrow \infty$ to get the same conclusion for $U$.

Applying the Alexandrov symmetry principle. We fix two points $x$ and $x^{\prime}$ in $\mathbb{R}^{N}$ such that $|x|<\left|x^{\prime}\right|$. Let $H$ denote the hyperplane perpendicular on the line $x x^{\prime}$. Let $\Omega_{1}$ and $\Omega_{2}$ be the two sets delimited by the hyperplane $H$ such that the origin is contained in $\Omega_{1}$. Let $\Pi$ the symmetry with respect to $H$ that maps $\Omega_{1}$ into $\Omega_{2}$. Clearly, $\Pi(x)=x^{\prime}, x \in \Omega_{1}$. Then one can prove that for every $y \in \Omega_{1}|y|<\left|y^{\prime}\right|$, where $y^{\prime}=\Pi(y)$. Since $v_{0 n}$ is radially nonincreasing, we get that $v_{0 n}(y) \geq v_{0 n}(\Pi(y))$ for all $y \in \Omega_{1}$. By applying the Alexandrov symmetry principle stated in Theorem 7.2 we obtain that $v_{n}(x) \geq v_{n}\left(x^{\prime}\right)$. The arguments we used can be done for every pair of points $|x|<\left|x^{\prime}\right|$; therefore $v_{n}$ is radially increasing.

II. Decay at infinity. This follows from the initial data of the solution $U$. In fact, fixing $x$ and letting $t \rightarrow 0$ we get $U(x, t) \rightarrow U_{0}(x)=C|x|^{\gamma}$ as $t \rightarrow 0$, which can be written as $t^{\gamma / 2 s} \mid F\left(t^{-1 / 2 s} x\right)-C\left(\left(t^{-1 / 2 s}|x|\right)^{\gamma} \mid \rightarrow 0\right.$ as $t \rightarrow 0$. In other words, $F(\eta) / \eta^{\gamma} \rightarrow C$ as $\eta \rightarrow \infty$. 
This characterization of the profile $F$ gives us the following spatial decay for $U(x, t)$ for large times:

$$
C_{2}|x|^{\gamma} \leq U(x, t) \leq C_{1}|x|^{\gamma} \quad \text { for large }|x| t^{-1 / 2 s} .
$$

Moreover, we will prove the following relation between $F^{\prime}$ and $F$ :

$$
\left|\gamma F(\eta)-\eta F^{\prime}(\eta)\right| \leq \eta^{\gamma} \quad \text { for large } \eta>0 .
$$

As a consequence we can characterize the derivative $U_{t}$ :

$$
\begin{gathered}
U_{t}(x, t)=t^{\alpha_{1}-1} \frac{1}{2 s}\left(\gamma F(\eta)-\eta F^{\prime}(\eta)\right), \quad \eta=t^{-1 / 2 s}|x|, \\
U_{t}(x, t) \sim t^{-1}|x|^{\gamma} \quad \text { for large values of } t^{-1 / 2 s}|x| .
\end{gathered}
$$

The first step will be to obtain a formula for the profile $F(\eta)$. Therefore

$$
\begin{aligned}
U(x, t) & =K_{s}(x, t) \star U_{0}(x)=t^{-\frac{N}{2 s}} \int_{\mathbb{R}^{N}} f\left(t^{-\frac{1}{2 s}}|x-y|\right)|y|^{\gamma} d y, \quad z=t^{-\frac{1}{2 s}} y, \\
& =t^{\frac{\gamma}{2 s}} \int_{\mathbb{R}^{N}} f\left(t^{-\frac{1}{2 s}} x-z\right)|z|^{\gamma} d z .
\end{aligned}
$$

Since $U(x, t)$ has the self-similar form (6.7), then

$$
F\left(t^{-\frac{1}{2 s}} x\right)=\int_{\mathbb{R}^{N}} f\left(t^{-\frac{1}{2 s}} x-z\right)|z|^{\gamma} d z=\left(f \star U_{0}\right)\left(t^{-\frac{1}{2 s}} x\right) \quad \forall x \in \mathbb{R}^{N}, t>0,
$$

that is,

$$
F(\eta)=\int_{\mathbb{R}^{N}} f(\eta-z)|z|^{\gamma} d z \quad \forall \eta \in \mathbb{R}^{N}
$$

Let us continue using the notation

$$
F(|\eta|)=F(\eta), \quad f(|\eta|)=f(\eta) .
$$

We fix $\eta \in \mathbb{R}^{N}$. Let $|\eta|=\bar{\eta}$ and $\eta=\bar{\eta} e$ for a vector $e \in \mathbb{R}^{N}$ with $|e|=1$. Then

$$
F(\bar{\eta})=\int_{\mathbb{R}^{N}} f(|z|)|\eta-z|^{\gamma} d z=\bar{\eta}^{N+\gamma} \int_{\mathbb{R}^{N}} f(|\bar{\eta} y|)|e-y|^{\gamma} d y, \quad z=\bar{\eta} y .
$$

We differentiate in $\bar{\eta}$

$$
F^{\prime}(\bar{\eta})=\bar{\eta}^{N+\gamma-1} \int_{\mathbb{R}^{N}}\left[(N+\gamma) f(|\bar{\eta} y|)+\bar{\eta} y f^{\prime}(|\bar{\eta} y|)\right]|e-y|^{\gamma} d y .
$$

Therefore

$$
\begin{aligned}
& \bar{\eta} F^{\prime}(\bar{\eta})-\gamma F(\bar{\eta})=\bar{\eta}^{N+\gamma} \int_{\mathbb{R}^{N}}\left[N f(|\bar{\eta} y|)+\bar{\eta} y f^{\prime}(|\bar{\eta} y|)\right]|e-y|^{\gamma} d y, \quad z=\bar{\eta} y, \\
& \bar{\eta} F^{\prime}(\bar{\eta})-\gamma F(\bar{\eta})=\bar{\eta}^{\gamma} \int_{\mathbb{R}^{N}}\left[N f(|z|)+z f^{\prime}(|z|)\right]\left|e-\frac{z}{\bar{\eta}}\right|^{\gamma} d z
\end{aligned}
$$

Copyright (c) by SIAM. Unauthorized reproduction of this article is prohibited. 
We know that $N f(r)+r f^{\prime}(r) \sim-C_{1} r^{-(N+2 s)}$ for large $r$. Since we deal with a convolution we will use the information only in the sense of modulus. We fix $R>0$ such that

$$
C_{1} r^{-(N+2 s)} \leq\left|N f(r)+z f^{\prime}(r)\right| \leq C_{2} r^{-(N+2 s)} \quad \forall r \geq R .
$$

The values of $R, C_{1}, C_{2}$ depend on the profile $f$ of the heat kernel $K_{s}(x, t)$. They are independent of the variable $\eta$ used in this proof. Then,

$$
\bar{\eta}^{-\gamma}\left|\bar{\eta} F^{\prime}(\bar{\eta})-\gamma F(\bar{\eta})\right| \leq \int_{\mathbb{R}^{N}}\left|N f(|z|)+z f^{\prime}(|z|)\right| \cdot\left|e-\frac{z}{\bar{\eta}}\right|^{\gamma} d z=\mathrm{I}+\mathrm{II},
$$

where

$$
\begin{aligned}
\mathrm{I} & =\int_{|z| \leq R}\left|N f(|z|)+z f^{\prime}(|z|)\right| \cdot\left|e-\frac{z}{\bar{\eta}}\right|^{\gamma} d z \\
& \leq C \int_{|z| \leq R}\left|e-\frac{z}{\bar{\eta}}\right|^{\gamma} d z \leq C\left(1+\frac{R}{\bar{\eta}}\right)^{\gamma} R^{N} .
\end{aligned}
$$

The second term is estimated as follows:

$$
\begin{aligned}
\mathrm{II} & =\int_{|z| \geq R}\left|N f(|z|)+z f^{\prime}(|z|)\right| \cdot\left|e-\frac{z}{\bar{\eta}}\right|^{\gamma} d z \leq C_{2} \int_{|z| \geq R}|z|^{-(N+2 s)}\left|e-\frac{z}{\bar{\eta}}\right|^{\gamma} d z \\
& \leq C_{2} \int_{|z| \geq R}|z|^{-(N+2 s)}\left(1+\frac{|z|}{\bar{\eta}}\right)^{\gamma} \leq C_{2} \int_{|z| \geq R}|z|^{-(N+2 s)}\left(2^{\gamma}+(2|z| / \bar{\eta})^{\gamma}\right) d z, \\
& =2^{\gamma} C_{2} \int_{|z| \geq R}|z|^{-(N+2 s)} d z+2^{\gamma} C_{2} \bar{\eta}^{-\gamma} \int_{|z| \geq R}|z|^{-(N+2 s)+\gamma} d z, \quad \text { we know } \gamma<2 s, \\
& =C_{3} R^{-2 s}+C_{3} \frac{1}{\bar{\eta}^{\gamma}} R^{\gamma-2 s}, \quad \text { where } \quad C_{3}=C_{3}\left(C_{2}, \gamma, \operatorname{meas}\left(\partial B_{1}\right)\right)>0 .
\end{aligned}
$$

We conclude that

$$
\mathrm{I}+\mathrm{II} \leq C_{4}+C_{5} \frac{1}{\bar{\eta}^{\gamma}},
$$

where the constants $C_{4}$ and $C_{5}$ depend on $R, \gamma, C_{1}, C_{2}$. Now, recall that $\left|\bar{\eta} F^{\prime}(\bar{\eta})-\gamma F(\bar{\eta})\right| \leq \bar{\eta}^{\gamma}(\mathrm{I}+\mathrm{II})$. Therefore we have proved that

$$
\bar{\eta}^{-\gamma}\left|\bar{\eta} F^{\prime}(\bar{\eta})-\gamma F(\bar{\eta})\right| \leq C_{4}+C_{5} \quad \text { for large } \bar{\eta} \geq 1 .
$$

\section{Appendix.}

7.1. Concept of solution to problem (1.1). According to [14] there exists a unique mild solution of problem (2.1) corresponding to the initial datum $u_{0} \in L^{1}\left(\mathbb{R}^{N}\right)$, $0 \leq u_{0} \leq 1$, constructed by means of the tools of semigroup theory. Moreover, such $u$ is in fact a strong solution of the equation. In the case $m>1$, the $C^{\alpha}$ regularity of the solution follows from [5], and this has been extended to $m<1$ up to the extinction time (if there is one). Quantitative estimates of positivity of the solution for any $m>0$ corresponding to nonnegative data have been proved in [9]. Recently, the classical regularity of strong solutions was proved in [15].

As a consequence one obtains by rather standard methods the existence, uniqueness, and regularity properties of the solution to problem (1.1) corresponding to the 
initial datum $u_{0} \in L^{1}\left(\mathbb{R}^{N}\right), 0 \leq u_{0} \leq 1$. In order to prove the existence of a solution of the problem $u_{t}+L_{s} u^{m}=f(u)$, we use that $f$ is Lipschitz and the semigroup approach of the diffusion equation $\underline{u}_{t}+L_{s} \underline{u}^{m}=0$. Standard properties, like the maximum principle, hold also in our setting.

A more detailed analysis of these properties is beyond the purpose of this work.

7.2. A version of the maximum principle. We need an interesting version of the maximum principle proved by Cabré and Roquejoffre in [12, Lemma 2.9], suitable for comparisons in which fractional Laplacian operators are involved.

For $0 \leq \gamma<2 s$ we consider functions $v: \mathbb{R}^{N} \rightarrow \mathbb{R}$ such that

$$
\begin{gathered}
|v(x)| \leq C\left(1+|x|^{\gamma}\right) \text { in } \mathbb{R}^{N} \text { for some constant } C, \\
\left\{\begin{array}{l}
\text { for every } \epsilon>0 \text { there exists } \delta>0 \text { such that } \\
\text { if } x \in \mathbb{R}^{N} \text { and }|z| \leq \delta \text {, then }|u(x+z)-u(x)| \leq \epsilon\left(1+|x|^{\gamma}\right) .
\end{array}\right.
\end{gathered}
$$

We define $X_{\gamma}=\left\{u: \mathbb{R}^{N} \rightarrow \mathbb{R}: u\right.$ satisfies (7.1) and (7.2) $\}$ and $D_{\gamma}$ is the domain of the operator $L_{s}=(-\Delta)^{s}$ in $X_{\gamma}$.

Lemma 7.1. Let $N \geq 1, s \in(0,1), 0 \leq \gamma<2 s$. Let $v \in C^{1}\left([0, \infty) ; X_{\gamma}\right)$ satisfy $v(\cdot, t) \in D_{\gamma}$ for all $t>0$. Let $r:(0, \infty) \rightarrow(0, \infty)$ be a continuous function and define

$$
\Omega_{I}=\left\{(x, t) \in \mathbb{R}^{N} \times(0, \infty):|x|<r(t)\right\} .
$$

Assume in addition the following:

(H1) $v(\cdot, 0) \leq 0$ in $\mathbb{R}^{N}$.

(H2) $v \leq 0$ in $\left(\mathbb{R}^{N} \times(0, \infty)\right) \backslash \Omega_{I}$.

(H3) $a(x, t) v_{t}+L_{s} v \leq b v$ in $\Omega_{I}$.

Then $v \leq 0$ in $\mathbb{R}^{N} \times(0, \infty)$.

Although the equation we have is different, the proof as in [12] still works (with inessential modifications).

7.3. Alexandrov reflection principle. We recall the version of Alexandrov's symmetry principle that holds in the case of the nonlinear parabolic problem

$$
u_{t}=L_{s} u^{m}, \quad u(0, x)=u_{0}(x),
$$

posed in $\mathbb{R}^{N}$, with $L_{s}=(-\Delta)^{s}, m>0, u_{0} \in L^{1}\left(\mathbb{R}^{N}\right)$. Let us take a hyperplane $H$ that divides $\mathbb{R}^{N}$ into two half-spaces $\Omega_{1}$ and $\Omega_{2}$ and consider the symmetry $\Pi$ with respect to $H$ that maps $\Omega_{1}$ into $\Omega_{2}$. The following result is proved as Theorem 15.2 in $[26]$.

THEOREM 7.2. Let $u$ be the unique solution of problem (7.3) with initial data $u_{0}$. Under the assumption that

$$
u_{0}(x) \geq u_{0}(\Pi(x)) \quad \text { in } \Omega_{1}
$$

we have that for all $t>0$

$$
u(x, t) \geq u(\Pi(x), t) \quad \text { for } x \in \Omega_{1} .
$$

7.4. Bessel functions of first kind. The Bessel function $J_{\mu}$ of first kind can be introduced through a series expansion (cf. [1]),

$$
J_{\mu}(z)=\sum_{k=0}^{\infty} \frac{(-1)^{k}}{k ! \Gamma(k+\mu+1)}\left(\frac{z}{2}\right)^{2 k+\mu} .
$$

Copyright $@$ by SIAM. Unauthorized reproduction of this article is prohibited. 
We mention the following recurrence formulas:

$$
\begin{aligned}
J_{\mu}^{\prime}(z) & =\frac{1}{2}\left(J_{\mu-1}(z)-J_{\mu+1}(z)\right) \quad \text { for } \mu \neq 0 . \\
J_{0}^{\prime}(z) & =-J_{1}(z), \\
J_{\mu}(z) & =\frac{z}{2 \mu}\left(J_{\mu-1}(z)+J_{\mu+1}(z)\right), \\
\int_{0}^{\infty} K_{a}(t) t^{b-1} d t & =2^{b-2} \Gamma\left(\frac{b+a}{2}\right) \Gamma\left(\frac{b-a}{2}\right), \quad \operatorname{Re}(b \pm a)>0 .
\end{aligned}
$$

The modified Hankel functions (cf. [18, p. 82]) are defined by

$$
K_{\nu}(z)=\int_{0}^{\infty} e^{-z \cosh t} \cosh (\nu t) d t \quad \text { for } \operatorname{Re}(z)>0 .
$$

Then $K_{\nu}(z) \in \mathbb{R}$ when $\nu \in \mathbb{R}$ is real and $z \in \mathbb{R}_{+}$. When $\nu=n+\frac{1}{2}, n \in \mathbb{N}$, then

$$
K_{n+1 / 2}(z)=\left(\frac{\pi}{2 z}\right)^{1 / 2} e^{-z} \sum_{m=0}^{n}(2 z)^{-m} \frac{\Gamma(n+m+1)}{m ! \Gamma(n+1-m)} .
$$

\section{Comments and open problems.}

- There are critical values of the speed $\sigma$ which we do not cover in this work: $\sigma_{1}$ for $m_{c}<m<m_{1} ; \sigma_{2}$ for $m_{1}<m \leq 1$; respectively, $\left(\sigma_{2}, \sigma_{3}\right)$ for $m>1$. The analysis of those cases leads to long new developments.

- Is there a definite profile function that represents up to translation the shape of the solution in the region where it varies in a marked way to join the level $u=1$ to the level $u=0$ ? Maybe for $s=1 / 2$ this question is easier.

- For reasons of length and novelty, the case $m<m_{c}$ is not studied. For the corresponding fractional fast diffusion equation there appears the phenomenon of extinction in finite time. King and McCabe in [22] give an idea on the asymptotics in this range of parameters.

- A detailed numerical treatment of these problems for the case $m \neq 1$ is needed; see in this respect [24].

- There are other interesting directions in this class of problems. Thus, in a recent paper [10], the authors investigate the model

$$
u_{t}(x, t)+A u(x, t)=\mu(x) u-u^{2}, \quad x \in \mathbb{R}^{N}, t>0,
$$

where the function $\mu$ is supposed periodic in each spatial variable $x_{i}$ and satisfy $0<\min \mu \leq \mu(x)$.

- Initial data with slow decay. The decay of the initial data plays an important role in the propagation of level sets. The slower the decay, the faster the propagation. We comment on some recent work on the issue:

- In [21], Hamel and Roques consider the one-dimensional classical Fisher-KPP problem $u_{t}=u_{x x}+f(u)$ with initial data $u_{0}$ that are assumed to decay at infinity more slowly than any exponentially decaying function. A precise quantitative estimate of the level sets of the solution is obtained in terms of the decay of the initial data, and this implies an exponential rate of propagation of level sets.

- As for the Fisher-KPP with fractional diffusion, the case of slowly decaying initial conditions has been recently treated by Felmer and Yangari in [19] for 
the linear case $u_{t}+L_{s} u=f(u)$. Assuming the initial data satisfy $u_{0}(x) \geq$ $|x|^{-b}$ with $b \in(0,2 s)$, they prove that the level sets of the solution propagate exponentially with a faster speed, thus completing the case studied by Hamel and Roques to all $s \in(0,1)$.

As far as we know, slowly decaying initial data have not been considered for nonlinear fractional diffusion cases. For our model, the proof of the convergence to 1 still works, since the pure diffusion problem, whose solutions are subsolutions for problem (1.1), reaches a tail-type behavior at a larger time. As for the convergence to 0 in the far field, we mention that our proof does not adapt to slower decay data since the main technique is using the long tail behavior of the Barenblatt solution.

Acknowledgment. We thank the anonymous referees for useful comments that resulted in a real improvement of the presentation.

\section{REFERENCES}

[1] M. Abramowitz And I. A. Stegun, Handbook of Mathematical Functions with Formulas, Graphs, and Mathematical Tables, National Bureau of Standards Appl. Math. Ser. 55, U.S. Government Printing Office, Washington, DC, 1964.

[2] D. Applebaum, Lévy Processes and Stochastic Calculus, 2nd ed., Cambridge Stud. Adv. Math. 116, Cambridge University Press, Cambridge, UK, 2009.

[3] D. G. Aronson And H. F. Weinberger, Nonlinear diffusion in population genetics, combustion, and nerve pulse propagation, in Partial Differential Equations and Related Topics, Lecture Notes in Math. 446, Springer, Berlin, 1975, 5-49.

[4] D. G. Aronson and H. F. Weinberger, Multidimensional nonlinear diffusion arising in population genetics, Adv. Math., 30 (1978), pp. 33-76.

[5] I. Athanasopoulos and L. A. Caffareldi, Continuity of the temperature in boundary heat control problems, Adv. Math., 224 (2010), pp. 293-315.

[6] H. Berestycki, J. M. Roquejoffre, and L. Rossi, The periodic patch model for population dynamics with fractional diffusion, Discrete Contin. Dyn. Syst. Ser. S, 4 (2011), pp. 1-13.

[7] J. Bertoin, Lévy Processes, Cambridge Tracts in Math. 121, Cambridge University Press, Cambridge, UK, 1996.

[8] R. M. Blumenthal and R. K. Getoon, Some theorems on stable processes, Trans. Amer. Math. Soc., 95 (1960), pp. 263-273.

[9] M. Bonforte And J. L. VÁzQuez, Quantitative local and global a priori estimates for fractional nonlinear diffusion equations, Adv. Math., 250 (2014), pp. 242-284.

[10] X. Cabré, A. C. Coulon, and J. M. Roquejoffre, Propagation in Fisher-KPP type equations with fractional diffusion in periodic media, C. R. Math. Acad. Sci. Paris, 350 (2012), pp. 885-890.

[11] X. Cabré and J. M. Roquejoffre, Propagation de fronts dans les équations de Fisher-KPP avec diffusion fractionnaire, C. R. Math. Acad. Sci. Paris, 347 (2009), pp. 1361-1366.

[12] X. Cabré And J. M. Roquejoffre, Front propagation in Fisher-KPP equations with fractional diffusion, Comm. Math. Phys., 320 (2013), pp. 679-722.

[13] A. De Pablo, F. Quirós, A. Rodriguez, and J. L. VÁzquez, A fractional porous medium equation, Adv. Math., 226 (2011), pp. 1378-1409.

[14] A. De Pablo, F. Quirós, A. Rodriguez, and J. L. VÁzquez, A general fractional porous medium equation, Comm. Pure Appl. Math., 65 (2012), pp. 1242-1284.

[15] A. de Pablo, F. Quirós, A. Rodríguez, and J. L. VÁzquez, Classical Solutions and Higher Regularity for Nonlinear Fractional Diffusion Equations, arXiv:1311.7427, 2013.

[16] A. DE PABlo And A. SÁnchez, Travelling wave behaviour for a porous-Fisher equation, European J. Appl. Math., 9 (1998), pp. 285-304.

[17] A. de PAblo And J. L. VÁzquez, Travelling waves and finite propagation in a reactiondiffusion equation, J. Differential Equations, 93 (1991), pp. 19-61.

[18] A. Erdélyi, W. Magnus, F. Oberhettinger, and F. Tricomi, Higher Transcendental Functions, vol. II, Robert E. Krieger Publishing, Melbourne, FL, 1981.

[19] P. Felmer And M. Yangari, Fast propagation for fractional KPP equations with slowly decaying initial conditions, SIAM J. Math. Anal., 45 (2013), pp. 662-678.

[20] R. A. Fisher, The wave of advance of advantagenous genes, Ann. Eugenics, 7 (1937), pp. $355-369$. 
[21] F. Hamel And L. Roques, Fast propagation for KPP equations with slowly decaying initial conditions, J. Differential Equations, 249 (2010), pp. 1726-1745.

[22] J. King and P. MCCabe, On the Fisher-KPP equation with fast nonlinear diffusion, R. Soc. Lond. Proc. Ser. A Math. Phys. Eng. Sci., 459 (2003), pp. 2529-2546.

[23] A. N. Kolmogorov, I. G. Petrovskit, and N. S. Piskunov, Etude de l'équation de diffusion avec accroissement de la quantité de matière, et son application à un problème biologique, Bjul. Moskowskogo Gos. Univ., 17 (1937), pp. 1-26.

[24] F. DEL TESO, Finite difference method for a fractional porous medium equation, Calcolo, Dec. 2013.

[25] E. VAldinoci, From the long jump random walk to the fractional Laplacian, Bol. Soc. Esp. Mat. Apl., 49 (2009), pp. 33-44.

[26] J. L. VÁzQUEZ, Barenblatt solutions and asymptotic behaviour for a nonlinear fractional heat equation of porous medium type, J. Eur. Math. Soc. (JEMS), 16 (2014), pp. 769-803.

[27] J. L. VÁzQuez, Asymptotic behaviour for the porous medium equation posed in the whole space, J. Evol. Equ., 3 (2003), pp. 67-118.

[28] J. L. VÁzQuez ANd B. Volzone, Symmetrization for linear and nonlinear fractional parabolic equations of porous medium type, J. Math. Pures Appl. (9), 101 (2014), pp. 553-582.

Copyright $@$ by SIAM. Unauthorized reproduction of this article is prohibited. 